\title{
Burial and thermal maturity modeling of the Middle Cretaceous- Early Miocene petroleum system, Iranian sector of the Persian Gulf
}

\author{
Zahra Sadat Mashhadi ${ }^{1}$ - Ahmad Reza Rabbani ${ }^{1} \cdot$ Mohammad Reza Kamali $^{2}$ • \\ Maryam Mirshahani $^{2} \cdot$ Ahmad Khajehzadeh $^{2}$
}

Received: 10 February 2015/Published online: 21 July 2015

(c) The Author(s) 2015. This article is published with open access at Springerlink.com

\begin{abstract}
The Cretaceous Kazhdumi and Gurpi formations, Ahmadi Member of the Sarvak Formation, and Paleogene Pabdeh Formation are important source rock candidates of the Middle Cretaceous-Early Miocene petroleum system in the Persian Gulf. This study characterizes generation potential, type of organic matter, and thermal maturity of 262 cutting samples (marls and argillaceous limestones) from these rock units taken from 16 fields in the Iranian sector of the Persian Gulf. In addition, the burial and thermal histories of these source rocks were analyzed by one-dimensional basin modeling. Based on the total organic carbon and genetic potential values, fair hydrocarbon generation potential is suggested for the studied samples. Based on $T_{\max }$ and vitrinite reflectance values, the studied samples are thermally immature to mature for hydrocarbon generation. The generated models indicate that studied source rocks are immature in central wells. The Gurpi and Pabdeh formations are immature and the Ahmadi Member and Kazhdumi Formation are early mature in the western wells. The Pabdeh Formation is within the main oil window and other source rocks are at the late oil window in the eastern wells. The hydrocarbon expulsion from the source rocks began after deposition of related caprocks which ensures entrapment and preservation of migrated hydrocarbon.
\end{abstract}

Ahmad Reza Rabbani

rabbani@aut.ac.ir

1 Petroleum Engineering Department, Amirkabir University of Technology, Hafez Street, 15875-4413 Tehran, Iran

2 Research Institute of Petroleum Industry (RIPI), West Blvd. Azadi Sport Complex, 14665-37 Tehran, Iran

Edited by Jie Hao
Keywords Persian Gulf · Kazhdumi Formation · Ahmadi Member - Gurpi Formation · Pabdeh Formation - Middle Cretaceous-Early Miocene petroleum system

\section{Introduction}

The Persian Gulf and its coastal areas (Fig. 1) contain the largest occurrence of crude oil in the world (Haghi et al. 2013) accounting for two-thirds of the world's proven oil reserves and approximately more than one-third of total proven world gas reserves (Rabbani 2007). Existence of repeated and extensive source rock beds, substantial carbonate and some sandstone reservoirs, excellent regional caprocks, huge anticlinal traps, and continuous sedimentation are the major factors making this region a remarkable area for hydrocarbon accumulations (Rabbani 2008).

The Middle Cretaceous-Early Miocene petroleum system is one of the five petroleum systems of the Zagros foldbelt and the Persian Gulf area (Bordenave and Hegre 2010). The Oligo-Miocene Asmari and Cretaceous Bangestan are the main reservoirs and the Cretaceous Kazhdumi Formation, Ahmadi Member of the Sarvak Formation, Gurpi Formation, and Paleogene Pabdeh Formation are important source rock candidates of this petroleum system. The evaporites of the Gachsaran Formation are cap rocks of this petroleum system. The Kazhdumi and Pabdeh formations are excellent source rocks and the Ahmadi Member and Gurpi Formation have been identified as marginal source rocks in the Dezful embayment (Bordenave and Burwood 1990; Bordenave and Huc 1995; Bordenave 2002; Bordenave and Hegre 2010; Rabbani and Tirtashi 2010; Alizadeh et al. 2012; Opera et al. 2013).

Despite the significant hydrocarbon accumulation in the Middle Cretaceous-Early Miocene petroleum system 


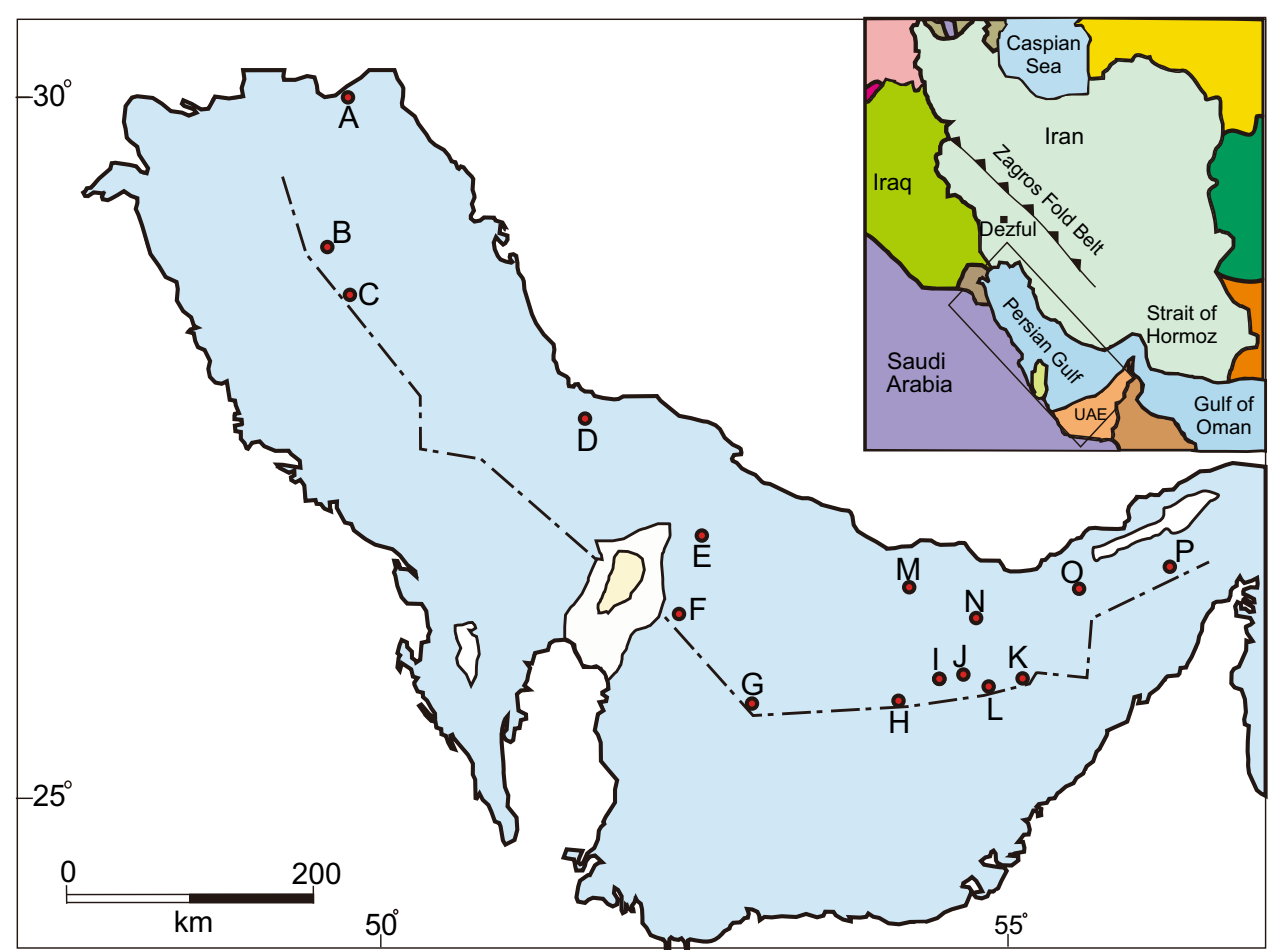

Fig. 1 Location of the studied fields in the Iranian sector of the Persian Gulf

within the Persian Gulf, little is known about the quality and maturity of the potential source rocks of this petroleum system in this area. This study tries to investigate hydrocarbon generation potential, depositional environment, and thermal maturity of the Kazhdumi Formation, Ahmadi Member, Gurpi Formation, and Pabdeh Formation in 16 fields located in the Iranian sector of the Persian Gulf (Fig. 1) by using Rock-Eval pyrolysis, molecular composition, and vitrinite reflectance measurement. Also, 1D basin modeling, a very useful tool in exploration-related studies, was applied to investigate the thermal maturity evolution and timing of hydrocarbon generation of these source rock candidates in the study area. The integration of the results of source rock characterization and basin modeling provides more detailed information to answer exploration questions. Accurate identification of a source rock helps to characterize the petroleum system and predict the location of future prospects charged by that source rock.

\section{Geological setting}

The Persian Gulf forms the northeast portion of the anticlockwise-moving Arabian Plate and formed during the Late Miocene (Alavi 2004). The Persian Gulf is situated at the junction of the Arabian and Eurasian lithospheric plates. It is structurally a foreland basin filled by terrigenous clastics transported from adjacent regions and carbonate sediments generated across the ramp surface (Ghazban 2009). Figure 2 shows the general lithostratigraphic column for the Iranian sector of the Persian Gulf.

During the Paleozoic, the Arabian Plate including the Persian Gulf region was located in the southern hemisphere with predominantly clastic sedimentation (Konert et al. 2001). Afterwards, during the Mesozoic and Cenozoic, the study area was mainly in tropical regions where carbonate deposition prevailed (Murris 1980; Ziegler 2001). Throughout most of the Mesozoic and up to the Lower Miocene, the area was part of a broad, shallow carbonate platform.

The Mesozoic carbonate systems of the Persian Gulf contain most of the extensive reservoir rocks in this area and form one of the richest hydrocarbon provinces in the world. This was mostly due to their vast-scale deposition and presence of source rocks, reservoir, and seal cap facies within the depositional system (Murris 1980). Within this time interval, in the Jurassic and Early Cretaceous periods, maximum marine transgression led to high production of organic matter, and its deposition under anoxic conditions forming organic rich deposits that were transformed over geologic time into petroleum source rocks (Alsharhan and Nairn 1997). Thick evaporites of the Gachsaran Formation, limestones and marls of the Mishan Formation followed by the sandstone, red marls, and siltstones of the Agha Jari Formation characterize the Mio-Pliocene of the region. 


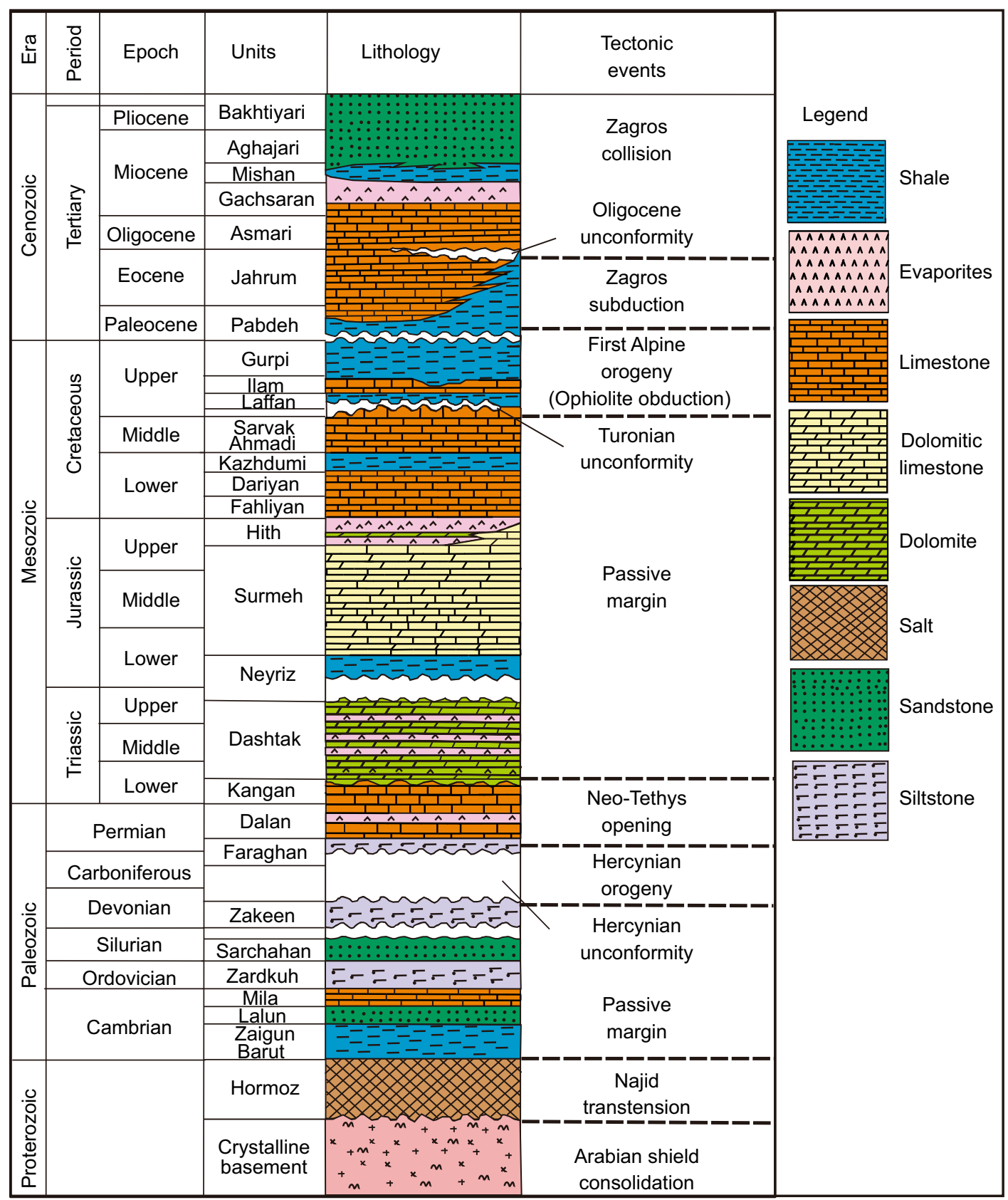

Fig. 2 Generalized stratigraphic column of Iranian sector of the Persian Gulf (modified from Al-Husseini 2008)

Folding accompanied by syntectonic and post-tectonic molasses took place in Plio-Pleistocene (Rabbani 2013).

The morphology of the Persian Gulf is highly affected by the Qatar Arch (Aali et al. 2006). The Qatar Arch is a first-order structure that was created in the central Persian Gulf following the tectonic movements during the Late Precambrian to Early Cambrian in the region (Fig. 3). It is a very large (over $100 \mathrm{~km}$ wide and $300 \mathrm{~km}$ long) regional gentle anticline (Ziegler 2001). According to offshore seismic data in the study area, this structure has a northeast-southwest direction in the Iranian sector of the Persian
Gulf and continues southwards to the Qatar peninsula (Perotti et al. 2011). As Fig. 4 demonstrates, the thicknesses of the Pabdeh, Gurpi and Kazhdumi formations, and Ahmadi Member significantly decrease toward the central parts of the study area with a noticeable thinning which can be due to the effect of the Qatar Arch Paleohigh during depositional time (Alsharhan and Nairn 1997).

Salt diapirism is another significant structural element in the Persian Gulf $(<5-20 \mathrm{~km}$ in size) piercing the stratigraphic sequences at different levels. Anticlines and domes have been induced by deep-seated salt pillows and salt 


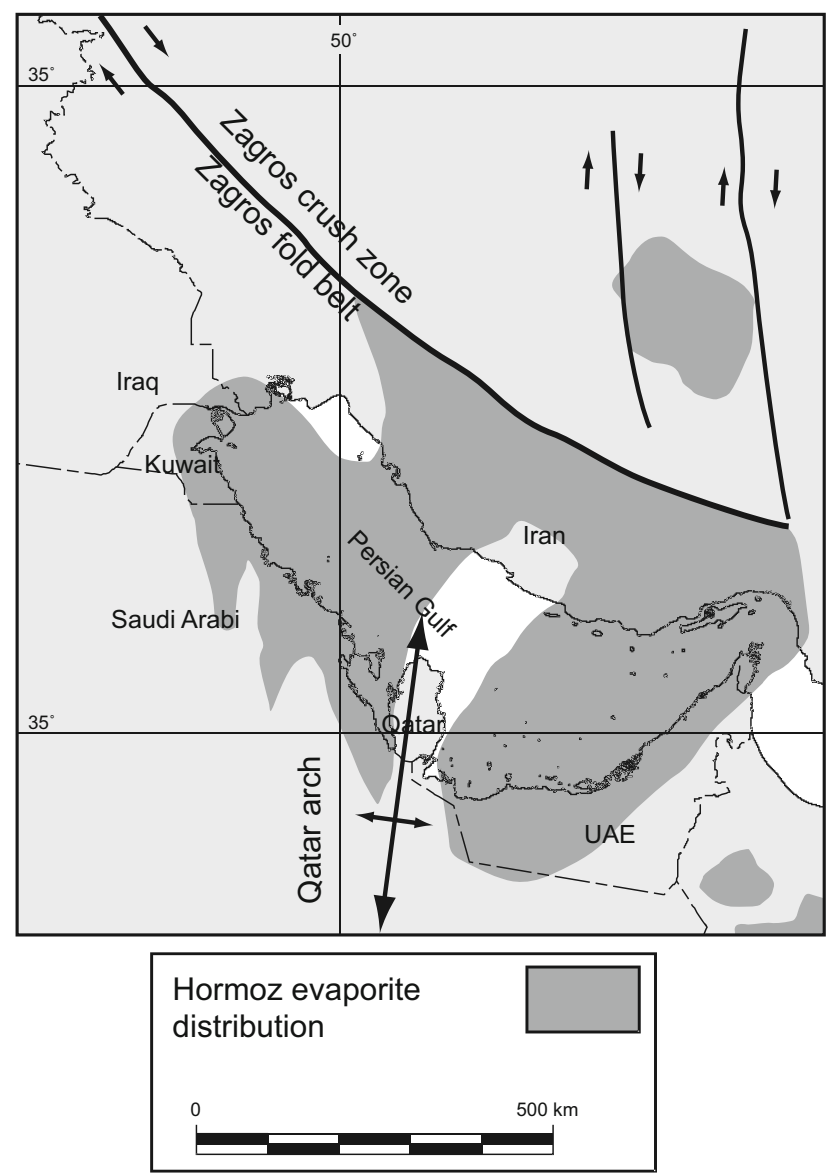

Fig. 3 Location of the Qatar Arch and distribution of Hormoz Salt in the study area (modified after Ghazban and Al-Aasm 2010)

ridges (Kent 1979). Almost all of this salt diapirism originates from the extrusion and remobilization of the InfraCambrian Hormoz Salt Series (Edgell 1991). Figure 3 shows the distribution of the Hormoz Salt in the Persian Gulf area.

\subsection{Kazhdumi Formation}

A transgression and sea-level rise in the Middle Cretaceous resulted in the deposition of the Kazhdumi Formation throughout the Albian (Alsharhan and Kendall 1991). In the Iranian offshore fields, the Kazhdumi Formation consists of calcareous shale and dark bituminous limestone with subordinate argillaceous limestone formed mostly in a neritic environment (Ghazban 2009). In addition, some thin sandstone beds may be present (Ghasemi-Nejad et al. 2009). The Burgan and Nahr Umr formations are the regional equivalents of the Kazhdumi Formation in the Arabian parts of the Persian Gulf (Rahmani et al. 2010). The underlying and overlying formations of the Kazhdumi
Formation are the Sarvak and Dariyan formations, respectively (Rahmani et al. 2013).

\subsection{Sarvak Formation}

The Sarvak Formation is part of the Bangestan Group and deposited as a result of a significant transgressive phase in the Middle Cretaceous, after regional emergence and periods of clastic and deltaic sedimentation (Alsharhan and Nairn 1997). The carbonates in the Sarvak Formation blanket most of the Persian Gulf area. The bituminous shaly limestone of the Mauddud and Khatiyah members (in the central and western parts of the Persian Gulf), the Ahmadi Member with shaly facies in the northern Persian Gulf, and the Mishrif reefal limestone member in the southern Persian Gulf (Ghazban 2009) are four members of the Sarvak Formation. The Laffan shales overlay the Sarvak Formation with an unconformity surface and act as an efficient regional seal for the Sarvak Reservoir. The Kazhdumi Formation underlies the Sarvak Formation with a transitional contact.

\subsection{Gurpi Formation}

The Gurpi Formation consists of thin bedded, deep-marine marl, and marly limestone deposited when local dysoxic conditions occurred in the northern Persian Gulf region. The Gurpi Formation can act as a seal for the Ilam reservoir which underlies the Gurpi Formation with an erosional disconformity (Homke et al. 2009). The Upper Aruma and Bahrah-Tayarat are the Gurpi equivalents in the coastal Arabia and Kuwait areas (Rabbani 2013).

\subsection{Pabdeh Formation}

Neritic to basinal marls and argillaceous limestones of the Pabdeh Formation deposited in a Paleocene-Eocene transgression which resulted from the Late Cretaceous tectonic activities. This formation consists of shale, marl, and argillaceous limestones (Soleimani et al. 2013). A monotonous deep-water shale facies with a limestone unit in its middle part is the main lithology of the Pabdeh Formation. Based on lithological characteristics, the Pabdeh represents deposition in a deep-water, anoxic environment in an overall transgressive sequence. In the northern Persian Gulf, the Asmari and Gurpi formations overlay and underlie the Pabdeh Formation, respectively. There appears to be a transition to clean limestones of the Jahrum Formation toward the southwest (Sharland 2001). Regional equivalents of the Pabdeh Formation are the Umm er Radhuma, Rus, and Dammam formations. 


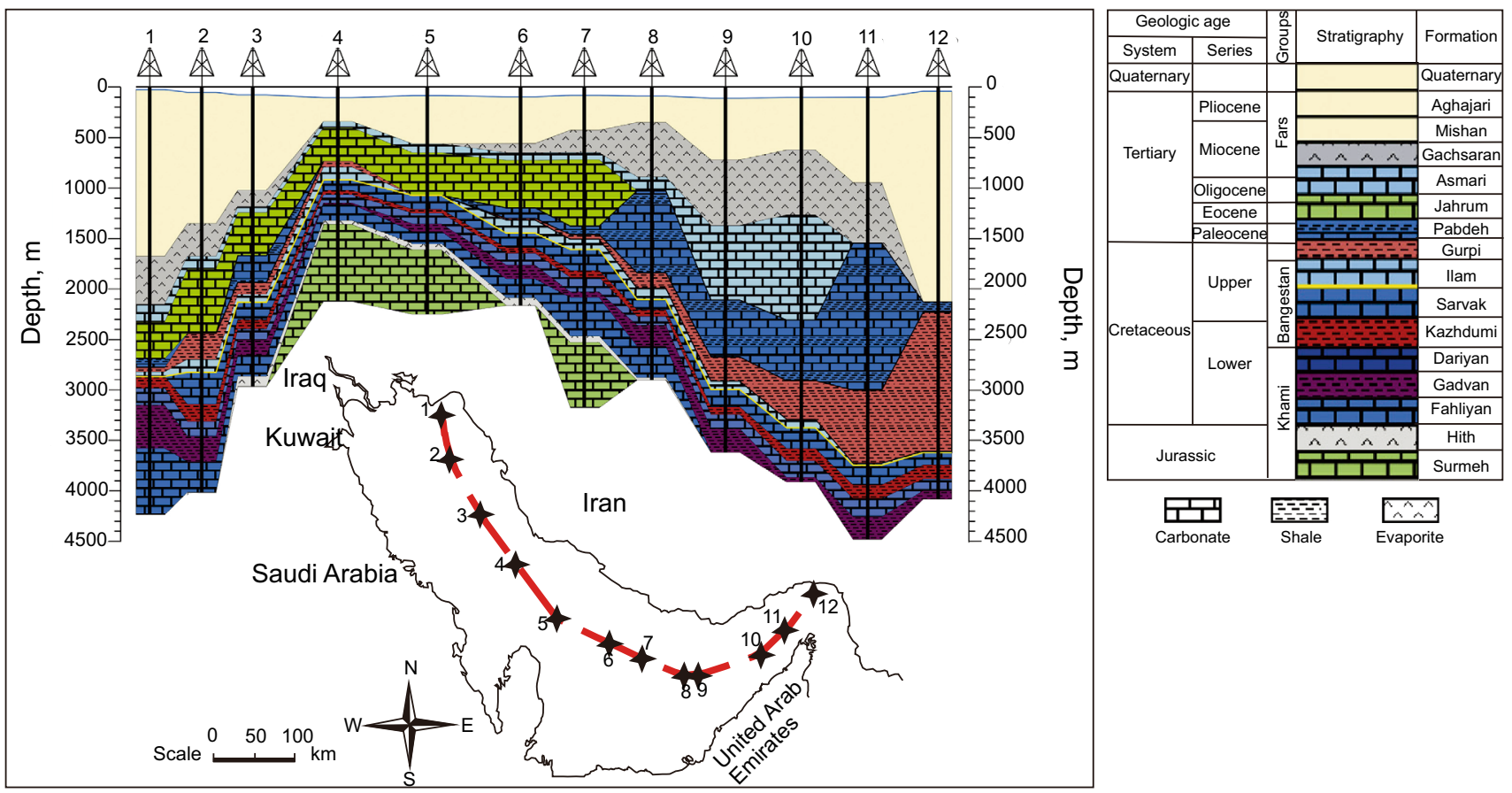

Fig. 4 Lithostratigraphic cross section in the Iranian sector of the Persian Gulf through Jurassic to Quaternary (modified after Rabbani et al. 2014)

\section{Materials and methods}

A total of 262 samples (including shale, marl, and argillaceous limestone) from the Kazhdumi, Gurpi, and Pabdeh formations and Ahmadi Member were taken from 16 fields within the Iranian sector of the Persian Gulf (Tables 1, 2, 3, 4, 5; Fig. 1). The selected samples were washed with water and detergent several times to remove contaminants from drilling mud additives. Then, the samples were crushed, pulverized, and homogenized. RockEval pyrolysis was performed by a Vinci Rock-Eval 6 instrument in the AGH University of Poland on a $50 \mathrm{mg}$ rock sample. See Espitalié et al. (1977), Lafargue et al. (1998) and Peters et al. (2005) for the details of this method. After completing the pyrolysis, the samples were heated to $850{ }^{\circ} \mathrm{C}$ at a rate of $25^{\circ} \mathrm{C} / \mathrm{min}$ in an oxidation oven and in the presence of air to oxidize (burn) all of the residual carbon. This process generates $\mathrm{CO}$ and $\mathrm{CO}_{2}$ which are measured quantitatively. The parameters measured by this analysis included the total organic carbon (TOC) (wt $\%$ ), $S_{1}$ (mg HC/g rock), $S_{2}$ (mg HC/g rock), $T_{\max }$ (the temperature at which the $S_{2}$ peak is the highest, ${ }^{\circ} \mathrm{C}$ ), and $S_{3}$ (mg $\mathrm{CO}_{2} / \mathrm{g}$ rock). Moreover, the hydrogen index (HI), oxygen index $(\mathrm{OI})$, production index $(\mathrm{PI})$, and migration index $\left(S_{1} / \mathrm{TOC}\right)$ were calculated.

Bitumen extractions were performed on approximately $10-15 \mathrm{~g}$ of 18 powdered samples from the Kazhdumi,
Gurpi, and Pabdeh formations and also Ahmadi Member by using a Soxhlet apparatus for $72 \mathrm{~h}$ with an azeotropic mixture of dichloromethane (DCM) and methanol $\left(\mathrm{CH}_{3} \mathrm{OH}\right)$ (93:7). The analyzed samples were selected based on higher organic matter content. The extracted bitumen in the rock samples was deasphalted by precipitation with $n$-hexane. Aliphatic, aromatic, and polar fractions were separated from the deasphalted samples by liquid column chromatography. The saturated fractions in the extracted bitumens were analyzed by gas chromatography (GC) in the AGH University of Poland. A Hewlett Packard 5890 series II GC held at a temperature of $300{ }^{\circ} \mathrm{C}$ equipped with a $50 \mathrm{~m} \times 0.2 \mathrm{~mm}$ Agilent DB1 column ( $0.5 \mu \mathrm{m}$ film thickness) with a constant flow of $0.3 \mathrm{~mL} / \mathrm{min}$ of nitrogen as a carrier gas was used for this analysis. The column oven was programmed to hold at $30{ }^{\circ} \mathrm{C}$ for $5 \mathrm{~min}$ and then increase to $320{ }^{\circ} \mathrm{C}$ at a rate of $3{ }^{\circ} \mathrm{C} / \mathrm{min}$. The oven stays at $320^{\circ} \mathrm{C}$ for $20 \mathrm{~min}$. The components eluting the column were detected by a flame ionization detector (FID) held at $325{ }^{\circ} \mathrm{C}$.

The vitrinite reflectance was measured in a random mode according to Taylor et al. (1998) and reported in $R_{\mathrm{o}} \%$. Based on the amount of organic carbon present within the samples, 50 samples were selected for this analysis. The samples were mounted in resin, and then ground and polished using an alumina-ethanol slurry. The analysis was carried out with a Leitz-MPV-SP microscope 
Table 1 Rock-Eval and vitrinite reflectance data for the Pabdeh samples

\begin{tabular}{|c|c|c|c|c|c|c|c|c|c|c|c|c|c|}
\hline Field & Well & Lith. $^{\mathrm{a}}$ & $\mathrm{MD}, \mathrm{m}$ & TOC & $T_{\max }$ & $S_{1}$ & $S_{1} / \mathrm{TOC}$ & $S_{2}$ & $S_{1}+S_{2}$ & $S_{3}$ & PI & HI & $R_{\mathrm{o}}, \%$ \\
\hline \multirow[t]{6}{*}{ M } & \multirow[t]{2}{*}{ M-1 } & ShMl & 1341 & 0.37 & 436 & 0.52 & 1.41 & 0.65 & 1.17 & 0.87 & 0.44 & 176 & \\
\hline & & ShMl & 1320 & 0.43 & 432 & 0.40 & 0.93 & 0.52 & 0.92 & 0.97 & 0.43 & 121 & 0.35 \\
\hline & \multirow[t]{4}{*}{ M-2 } & ShMl & 1159 & 1.59 & 430 & 0.11 & 0.07 & 2.92 & 3.03 & 1.24 & 0.04 & 184 & 0.35 \\
\hline & & ShMl & 1195 & 1.36 & 429 & 0.12 & 0.09 & 2.61 & 2.73 & 1.13 & 0.04 & 192 & \\
\hline & & ShMl & 1242 & 1.25 & 430 & 0.17 & 0.14 & 1.11 & 1.28 & 1.26 & 0.13 & & \\
\hline & & ShMl & 1280 & 1.33 & 431 & 0.10 & 0.08 & 1.46 & 1.56 & 1.37 & 0.06 & 110 & \\
\hline \multirow[t]{24}{*}{ I } & \multirow[t]{23}{*}{$\mathrm{I}-1$} & Ml & 2365 & 0.50 & 435 & 0.12 & 0.24 & 1.36 & 1.48 & 1.65 & 0.08 & 272 & \\
\hline & & Ml & 2355 & 0.43 & 433 & 0.15 & 0.35 & 1.37 & 1.52 & 1.71 & 0.10 & 319 & \\
\hline & & Ml & 2345 & 0.31 & 435 & 0.10 & 0.32 & 0.54 & 0.64 & 1.33 & 0.16 & 174 & \\
\hline & & ShMl & 2195 & 0.33 & 435 & 0.10 & 0.30 & 0.75 & 0.85 & 1.50 & 0.12 & 227 & \\
\hline & & Ml & 2165 & 0.37 & 434 & 0.12 & 0.32 & 1.08 & 1.20 & 1.54 & 0.10 & 292 & \\
\hline & & Ml & 2135 & 0.33 & 434 & 0.19 & 0.58 & 1.39 & 1.58 & 1.15 & 0.12 & 421 & \\
\hline & & Ml & 2100 & 0.39 & 433 & 0.12 & 0.31 & 1.47 & 1.59 & 1.34 & 0.08 & 377 & \\
\hline & & LimMl & 2080 & 0.34 & 434 & 0.10 & 0.29 & 0.97 & 1.07 & 1.36 & 0.09 & 285 & \\
\hline & & LimMl & 1955 & 0.55 & 432 & 0.12 & 0.22 & 2.89 & 3.01 & 1.09 & 0.04 & 525 & \\
\hline & & Ml & 1945 & 0.59 & 435 & 0.17 & 0.29 & 2.77 & 2.94 & 1.08 & 0.06 & 469 & \\
\hline & & Ml & 1895 & 0.52 & 433 & 0.12 & 0.23 & 2.24 & 2.36 & 0.83 & 0.05 & 431 & \\
\hline & & Ml & 1835 & 0.58 & 433 & 0.16 & 0.28 & 2.71 & 2.87 & 1.02 & 0.06 & 467 & \\
\hline & & Ml & 1825 & 0.62 & 438 & 0.19 & 0.31 & 2.61 & 2.80 & 0.96 & 0.07 & 421 & \\
\hline & & Ml & 1805 & 0.52 & 431 & 0.10 & 0.19 & 1.87 & 1.97 & 1.10 & 0.05 & 360 & \\
\hline & & Ml & 1795 & 0.46 & 434 & 0.13 & 0.28 & 1.60 & 1.73 & 1.17 & 0.08 & 348 & \\
\hline & & Ml & 1775 & 0.33 & 432 & 0.11 & 0.33 & 1.19 & 1.30 & 0.92 & 0.08 & 361 & \\
\hline & & Ml & 1765 & 0.51 & 434 & 0.17 & 0.33 & 1.57 & 1.74 & 0.67 & 0.10 & 308 & \\
\hline & & Ml & 1755 & 0.63 & 431 & 0.18 & 0.29 & 1.20 & 1.38 & 1.15 & 0.13 & 190 & \\
\hline & & Ml & 1725 & 0.31 & 434 & 0.11 & 0.35 & 0.97 & 1.08 & 0.86 & 0.10 & 313 & \\
\hline & & Ml & 1705 & 0.33 & 430 & 0.10 & 0.30 & 0.96 & 1.06 & 0.89 & 0.09 & 291 & \\
\hline & & Ml & 1695 & 0.45 & 433 & 0.08 & 0.18 & 0.81 & 0.89 & 1.17 & 0.09 & 180 & \\
\hline & & LimMl & 1625 & 0.33 & 436 & 0.14 & 0.42 & 0.93 & 1.07 & 1.82 & 0.14 & 282 & \\
\hline & & Ml & 1305 & 0.31 & 428 & 0.16 & 0.52 & 1.18 & 1.34 & 0.78 & 0.12 & 381 & \\
\hline & $\mathrm{I}-2$ & Ml & 1763 & 0.38 & 439 & 0.14 & 0.37 & 0.62 & 0.76 & 0.70 & 0.18 & 163 & \\
\hline \multirow[t]{10}{*}{ K } & \multirow[t]{10}{*}{$\mathrm{K}-1$} & Ml & 2875 & 0.35 & 430 & 0.13 & 0.37 & 0.68 & 0.81 & 1.79 & 0.16 & 194 & \\
\hline & & Ml & 2865 & 0.40 & 433 & 0.11 & 0.28 & 0.86 & 0.97 & 1.25 & 0.12 & 215 & \\
\hline & & Ml & 2715 & 0.96 & 425 & 0.21 & 0.22 & 5.20 & 5.41 & 1.43 & 0.04 & 542 & \\
\hline & & Ml & 2485 & 0.53 & 427 & 0.11 & 0.21 & 1.34 & 1.45 & 1.71 & 0.07 & 253 & \\
\hline & & Ml & 2485 & 0.84 & 433 & 0.14 & 0.17 & 2.38 & 2.52 & 1.91 & 0.06 & 283 & \\
\hline & & Ml & 2455 & 1.31 & 433 & 0.23 & 0.18 & 4.54 & 4.77 & 1.77 & 0.05 & 347 & \\
\hline & & Ml & 2405 & 1.44 & 431 & 0.26 & 0.18 & 5.86 & 6.12 & 1.57 & 0.04 & 407 & \\
\hline & & Ml & 2375 & 1.50 & 430 & 0.27 & 0.18 & 8.91 & 9.18 & 1.66 & 0.03 & 594 & 0.45 \\
\hline & & Ml & 2298 & 1.04 & 434 & 0.26 & 0.25 & 4.69 & 4.95 & 1.40 & 0.05 & 451 & \\
\hline & & Ml & 2260 & 0.74 & 428 & 0.20 & 0.27 & 2.96 & 3.16 & 1.27 & 0.06 & 400 & \\
\hline \multirow[t]{2}{*}{$\mathrm{J}$} & \multirow[t]{2}{*}{$\mathrm{J}-1$} & MlSh & 2046 & 0.39 & 432 & 0.14 & 0.36 & 0.50 & 0.64 & 1.06 & 0.22 & & \\
\hline & & MlSh & 2040 & 0.71 & 434 & 0.29 & 0.41 & 1.89 & 2.18 & 1.36 & 0.13 & & \\
\hline \multirow[t]{3}{*}{$\mathrm{P}$} & \multirow[t]{3}{*}{ P-3 } & Ml & 2344 & 0.43 & 440 & 0.16 & 0.37 & 0.57 & 0.73 & 1.46 & 0.22 & 132 & \\
\hline & & Ml & 2329 & 0.34 & & 0.15 & 0.44 & 0.51 & 0.66 & 1.45 & 0.23 & 149 & \\
\hline & & Ml & 2316 & 0.45 & 433 & 0.17 & 0.38 & 0.55 & 0.72 & 1.47 & 0.24 & 122 & \\
\hline
\end{tabular}


Table 1 continued

\begin{tabular}{|c|c|c|c|c|c|c|c|c|c|c|c|c|c|}
\hline Field & Well & Lith. $^{\mathrm{a}}$ & $\mathrm{MD}, \mathrm{m}$ & TOC & $T_{\max }$ & $S_{1}$ & $S_{1} / \mathrm{TOC}$ & $S_{2}$ & $S_{1}+S_{2}$ & $S_{3}$ & $\mathrm{PI}$ & $\mathrm{HI}$ & $R_{\mathrm{o}}, \%$ \\
\hline & & Ml & 2310 & 0.33 & 431 & 0.16 & 0.49 & 0.50 & 0.66 & 0.96 & 0.24 & 152 & 0.75 \\
\hline & & Ml & 2272 & 0.37 & 431 & 0.17 & 0.46 & 1.33 & 1.50 & 1.75 & 0.11 & 363 & \\
\hline & & Ml & 2268 & 0.33 & 433 & 0.18 & 0.55 & 0.51 & 0.69 & 1.16 & 0.26 & 155 & \\
\hline & & Ml & 2264 & 0.36 & 434 & 0.19 & 0.52 & 0.87 & 1.06 & 1.49 & 0.18 & 240 & 0.68 \\
\hline & & Ml & 2252 & 0.36 & 430 & 0.17 & 0.47 & 0.62 & 0.79 & 0.96 & 0.22 & 172 & \\
\hline & & Ml & 2242 & 0.43 & 431 & 0.19 & 0.44 & 1.10 & 1.29 & 1.18 & 0.15 & 257 & \\
\hline & & Ml & 2212 & 0.36 & 436 & 0.23 & 0.64 & 0.78 & 1.01 & 0.94 & 0.23 & 216 & \\
\hline & & Ml & 2204 & 0.37 & 431 & 0.18 & 0.49 & 0.61 & 0.79 & 1.50 & 0.23 & 164 & \\
\hline & & Ml & 2192 & 0.44 & 431 & 0.21 & 0.48 & 1.07 & 1.28 & 1.13 & 0.16 & 242 & \\
\hline & & Ml & 2182 & 0.42 & 432 & 0.24 & 0.57 & 0.72 & 0.96 & 1.35 & 0.25 & 172 & 0.70 \\
\hline & & Ml & 2172 & 0.48 & 436 & 0.21 & 0.44 & 0.93 & 1.14 & 1.21 & 0.18 & 193 & \\
\hline & & Ml & 2162 & 0.41 & 431 & 0.20 & 0.49 & 0.60 & 0.80 & 1.06 & 0.25 & 147 & \\
\hline & & Ml & 2152 & 0.61 & 430 & 0.29 & 0.47 & 1.45 & 1.74 & 1.36 & 0.17 & 237 & \\
\hline & & Ml & 2143 & 0.50 & 429 & 0.21 & 0.42 & 0.70 & 0.91 & 1.21 & 0.23 & 141 & \\
\hline & & Ml & 2132 & 0.45 & 418 & 0.30 & 0.67 & 1.29 & 1.59 & 1.40 & 0.19 & 287 & 0.63 \\
\hline & & Ml & 2113 & 0.43 & 429 & 0.23 & 0.53 & 0.56 & 0.79 & 0.94 & 0.29 & 130 & \\
\hline & & Ml & 2106 & 0.35 & 434 & 0.21 & 0.60 & 0.62 & 0.83 & 1.02 & 0.25 & 178 & \\
\hline & & Ml & 2090 & 0.30 & 430 & 0.18 & 0.60 & 0.73 & 0.91 & 0.62 & 0.20 & 242 & 0.60 \\
\hline & & Ml & 2070 & 0.35 & & 0.16 & 0.46 & 0.52 & 0.68 & 0.65 & 0.24 & 149 & 0.62 \\
\hline & & Ml & 2054 & 0.47 & 438 & 0.15 & 0.32 & 0.57 & 0.72 & 0.49 & 0.21 & 122 & \\
\hline & & Ml & 2046 & 0.65 & 437 & 0.23 & 0.35 & 0.90 & 1.13 & 2.29 & 0.20 & 138 & \\
\hline & & Ml & 2034 & 0.45 & 433 & 0.22 & 0.49 & 0.82 & 1.04 & 1.88 & 0.21 & 184 & \\
\hline & & Ml & 2026 & 0.51 & 428 & 0.23 & 0.45 & 0.72 & 0.95 & 1.89 & 0.24 & 140 & \\
\hline & & Ml & 2014 & 0.71 & 420 & 0.30 & 0.42 & 1.19 & 1.49 & 1.46 & 0.20 & 168 & \\
\hline & & Ml & 2009 & 0.66 & 428 & 0.19 & 0.29 & 0.81 & 1.00 & 1.40 & 0.19 & 123 & \\
\hline & & Ml & 2005 & 0.62 & 426 & 0.21 & 0.34 & 0.90 & 1.11 & 1.17 & 0.19 & 145 & \\
\hline & & Ml & 1996 & 0.38 & 428 & 0.20 & 0.53 & 0.50 & 0.70 & 1.01 & 0.29 & 132 & \\
\hline & & Ml & 1994 & 0.70 & 433 & 0.29 & 0.41 & 1.12 & 1.41 & 3.35 & 0.21 & 160 & 0.63 \\
\hline & & Ml & 1986 & 0.81 & 428 & 0.27 & 0.33 & 1.32 & 1.59 & 1.83 & 0.17 & 162 & \\
\hline & & Ml & 1978 & 0.87 & 423 & 0.30 & 0.35 & 1.55 & 1.85 & 2.11 & 0.16 & 179 & \\
\hline & & Ml & 1970 & 0.75 & 426 & 0.29 & 0.39 & 1.30 & 1.59 & 1.77 & 0.18 & 174 & \\
\hline & & Ml & 1960 & 0.71 & 421 & 0.35 & 0.49 & 1.17 & 1.52 & 2.05 & 0.23 & 164 & \\
\hline & & Ml & 1946 & 0.56 & 426 & 0.27 & 0.49 & 0.74 & 1.01 & 1.89 & 0.27 & 133 & \\
\hline & & Ml & 1938 & 0.63 & 427 & 0.25 & 0.40 & 1.13 & 1.38 & 2.07 & 0.18 & 180 & \\
\hline & & Ml & 1930 & 0.59 & 428 & 0.25 & 0.42 & 0.91 & 1.16 & 2.15 & 0.22 & 153 & 0.58 \\
\hline & & Ml & 1922 & 0.75 & 426 & 0.22 & 0.29 & 1.16 & 1.38 & 1.52 & 0.16 & 155 & \\
\hline & & Ml & 1914 & 0.70 & 426 & 0.22 & 0.31 & 0.95 & 1.17 & 1.46 & 0.19 & 135 & \\
\hline & & Ml & 1898 & 0.46 & 433 & 0.19 & 0.41 & 0.73 & 0.92 & 1.38 & 0.21 & 158 & \\
\hline & & $\mathrm{Ml}$ & 1887 & 0.74 & 426 & 0.27 & 0.36 & 1.34 & 1.61 & 1.56 & 0.17 & 181 & \\
\hline & & Ml & 1878 & 0.76 & 426 & 0.28 & 0.37 & 1.18 & 1.46 & 1.61 & 0.19 & 156 & \\
\hline & & Ml & 1872 & 1.19 & 425 & 0.38 & 0.32 & 1.75 & 2.13 & 1.53 & 0.18 & 147 & 0.56 \\
\hline & & Ml & 1856 & 0.99 & 423 & 0.31 & 0.31 & 2.04 & 2.35 & 1.56 & 0.13 & 206 & \\
\hline & & Ml & 1846 & 0.98 & 426 & 0.31 & 0.32 & 1.52 & 1.83 & 1.97 & 0.17 & 155 & \\
\hline & & Ml & 1838 & 0.96 & 426 & 0.36 & 0.38 & 1.64 & 2.00 & 1.95 & 0.18 & 172 & \\
\hline & & Ml & 1819 & 0.93 & 425 & 0.34 & 0.37 & 1.45 & 1.79 & 1.93 & 0.19 & 156 & 0.54 \\
\hline & & Ml & 1808 & 0.83 & 427 & 0.41 & 0.50 & 1.48 & 1.89 & 1.63 & 0.22 & 179 & \\
\hline & & Ml & 1798 & 0.87 & 428 & 0.27 & 0.31 & 1.31 & 1.58 & 1.65 & 0.17 & 150 & \\
\hline & & Ml & 1788 & 0.87 & 436 & 0.26 & 0.30 & 1.18 & 1.44 & 1.50 & 0.18 & 135 & \\
\hline
\end{tabular}


Table 1 continued

\begin{tabular}{|c|c|c|c|c|c|c|c|c|c|c|c|c|c|}
\hline Field & Well & Lith. $^{\mathrm{a}}$ & $\mathrm{MD}, \mathrm{m}$ & TOC & $T_{\max }$ & $S_{1}$ & $S_{1} / \mathrm{TOC}$ & $S_{2}$ & $S_{1}+S_{2}$ & $S_{3}$ & PI & $\mathrm{HI}$ & $R_{\mathrm{o}}, \%$ \\
\hline & & Ml & 1773 & 1.27 & 428 & 0.32 & 0.25 & 2.11 & 2.43 & 1.86 & 0.13 & 166 & 0.48 \\
\hline & & Ml & 1736 & 1.49 & 431 & 0.40 & 0.27 & 2.66 & 3.06 & 1.69 & 0.13 & 179 & 0.46 \\
\hline & & Ml & 1728 & 1.47 & 430 & 0.38 & 0.26 & 2.71 & 3.09 & 1.79 & 0.12 & 184 & \\
\hline & & Ml & 1716 & 1.06 & 431 & 0.26 & 0.24 & 1.73 & 1.99 & 1.51 & 0.13 & 163 & \\
\hline & & Ml & 1702 & 1.25 & 429 & 0.22 & 0.18 & 2.16 & 2.38 & 1.60 & 0.09 & 173 & \\
\hline & & Ml & 1688 & 1.37 & 427 & 0.41 & 0.30 & 4.15 & 4.56 & 2.07 & 0.09 & 303 & \\
\hline & & Ml & 1676 & 0.99 & 430 & 0.26 & 0.26 & 1.91 & 2.17 & 1.80 & 0.12 & 194 & \\
\hline & & Ml & 1668 & 0.59 & 429 & 0.20 & 0.34 & 1.25 & 1.45 & 1.64 & 0.14 & 214 & 0.36 \\
\hline & & Ml & 1660 & 1.01 & 428 & 0.27 & 0.27 & 2.23 & 2.50 & 1.67 & 0.11 & 221 & \\
\hline & & Ml & 1652 & 0.67 & 428 & 0.31 & 0.46 & 1.60 & 1.91 & 1.71 & 0.16 & 239 & \\
\hline & & Ml & 1642 & 0.69 & 428 & 0.25 & 0.36 & 1.21 & 1.46 & 1.78 & 0.17 & 176 & \\
\hline & & Ml & 1632 & 0.83 & 428 & 0.32 & 0.39 & 1.88 & 2.20 & 1.67 & 0.15 & 227 & \\
\hline & & Ml & 1622 & 1.03 & 430 & 0.25 & 0.24 & 1.57 & 1.82 & 1.86 & 0.14 & 152 & 0.40 \\
\hline & & Ml & 1608 & 1.45 & 428 & 0.28 & 0.19 & 3.09 & 3.37 & 1.46 & 0.08 & 213 & \\
\hline & & Ml & 1596 & 1.56 & 427 & 0.27 & 0.17 & 3.12 & 3.39 & 1.95 & 0.08 & 200 & 0.48 \\
\hline & & Ml & 1588 & 1.58 & 425 & 0.43 & 0.27 & 4.57 & 5.00 & 1.65 & 0.09 & 290 & \\
\hline & & Ml & 1580 & 1.26 & 425 & 0.31 & 0.25 & 3.15 & 3.46 & 1.62 & 0.09 & 250 & \\
\hline & & Ml & 1572 & 1.66 & 427 & 0.68 & 0.41 & 5.15 & 5.83 & 1.76 & 0.12 & 310 & \\
\hline & & Ml & 1564 & 0.96 & 421 & 0.31 & 0.32 & 2.35 & 2.66 & 1.77 & 0.12 & 245 & 0.40 \\
\hline & & Ml & 1556 & 1.11 & 426 & 0.32 & 0.29 & 2.59 & 2.91 & 1.53 & 0.11 & 233 & \\
\hline & & Ml & 1544 & 1.07 & 416 & 0.40 & 0.38 & 2.59 & 2.99 & 1.78 & 0.13 & 243 & \\
\hline & & Ml & 1536 & 1.17 & 413 & 0.50 & 0.43 & 3.19 & 3.69 & 1.47 & 0.14 & 273 & \\
\hline & & Ml & 1528 & 0.98 & 404 & 0.36 & 0.37 & 2.33 & 2.69 & 1.35 & 0.13 & 237 & \\
\hline & & Ml & 1520 & 1.25 & 402 & 0.67 & 0.53 & 3.02 & 3.69 & 1.12 & 0.18 & 241 & \\
\hline & & Ml & 1512 & 1.50 & 410 & 0.66 & 0.44 & 3.56 & 4.22 & 1.59 & 0.16 & 238 & 0.55 \\
\hline \multirow[t]{17}{*}{$\mathrm{O}$} & \multirow[t]{17}{*}{$\mathrm{O}-2$} & Ml & 2372 & 1.11 & 408 & 0.80 & 0.72 & 3.60 & 4.40 & 1.78 & 0.18 & 324 & \\
\hline & & Ml & 2397 & 1.35 & 413 & 0.56 & 0.41 & 5.43 & 5.99 & 2.05 & 0.09 & 402 & \\
\hline & & Ml & 2423 & 1.91 & 423 & 0.59 & 0.31 & 7.66 & 8.25 & 2.10 & 0.07 & 401 & \\
\hline & & Ml & 2443 & 1.98 & 422 & 0.46 & 0.23 & 7.51 & 7.97 & 1.64 & 0.06 & 379 & \\
\hline & & Ml & 2463 & 1.43 & 421 & 0.57 & 0.40 & 6.09 & 6.66 & 1.92 & 0.09 & 426 & \\
\hline & & Ml & 2488 & 1.30 & 427 & 0.37 & 0.28 & 4.00 & 4.37 & 3.26 & 0.08 & 308 & \\
\hline & & Ml & 2507 & 1.99 & 421 & 0.40 & 0.20 & 8.20 & 8.60 & 2.19 & 0.05 & 412 & \\
\hline & & Ml & 2529 & 2.64 & 419 & 0.51 & 0.19 & 10.62 & 11.13 & 2.69 & 0.05 & 402 & \\
\hline & & Ml & 2550 & 3.36 & 419 & 0.63 & 0.19 & 15.55 & 16.18 & 1.68 & 0.04 & 463 & \\
\hline & & Ml & 2567 & 3.00 & 424 & 0.49 & 0.16 & 13.51 & 14.00 & 2.07 & 0.04 & 450 & \\
\hline & & Ml & 2591 & 2.95 & 424 & 0.51 & 0.17 & 13.37 & 13.88 & 1.56 & 0.04 & 453 & \\
\hline & & Ml & 2608 & 2.67 & 425 & 0.49 & 0.18 & 12.12 & 12.61 & 1.47 & 0.04 & 454 & 0.56 \\
\hline & & Ml & 2658 & 0.86 & 428 & 0.19 & 0.22 & 2.43 & 2.62 & 1.91 & 0.07 & 283 & \\
\hline & & Ml & 2688 & 1.01 & 429 & 0.18 & 0.18 & 2.06 & 2.24 & 2.40 & 0.08 & 204 & \\
\hline & & Ml & 2702 & 0.49 & 429 & 0.22 & 0.45 & 1.22 & 1.44 & 1.90 & 0.15 & 249 & \\
\hline & & Ml & 2722 & 0.55 & 427 & 0.15 & 0.27 & 0.80 & 0.95 & 2.27 & 0.16 & 145 & 0.58 \\
\hline & & Ml & 2748 & 3.77 & 424 & 0.54 & 0.14 & 16.39 & 16.93 & 2.00 & 0.03 & 435 & \\
\hline
\end{tabular}

The units of the Rock-Eval pyrolysis parameters and indices: TOC wt $\%, S_{1} \mathrm{mg} \mathrm{HC} / \mathrm{g}$ rock, $S_{2} \mathrm{mg} \mathrm{HC} / \mathrm{g}$ rock, $S_{1}+S_{2} \mathrm{mg} \mathrm{HC} / \mathrm{g}$ rock, $\mathrm{S}_{3} \mathrm{mg} \mathrm{CO} / \mathrm{g}$ rock, $T_{\max }{ }^{\circ} \mathrm{C}, \mathrm{HI} \mathrm{mg} \mathrm{HC} / \mathrm{g}$ TOC, OI $\mathrm{mg} \mathrm{CO}_{2} / \mathrm{g}$ TOC

a $\mathrm{ShMl}$ is shaly marl, $\mathrm{Ml}$ is marl, $\mathrm{Sh}$ is shale, MlSh is marly shale, and LiMl is limy marl 
Table 2 Rock-Eval and vitrinite reflectance data for the Gurpi samples

\begin{tabular}{|c|c|c|c|c|c|c|c|c|c|c|c|c|c|}
\hline Field & Well & Lith. ${ }^{a}$ & $\mathrm{MD}, \mathrm{m}$ & TOC & $T_{\max }$ & $S_{1}$ & $S_{1} / \mathrm{TOC}$ & $S_{2}$ & $S_{1}+S_{2}$ & $S_{3}$ & PI & $\mathrm{HI}$ & $R_{\mathrm{o}}, \%$ \\
\hline \multirow[t]{7}{*}{ M } & \multirow[t]{5}{*}{ M-1 } & ShMl & 1412 & 0.39 & 427 & 0.32 & 0.82 & 1.02 & 1.34 & 1.07 & 0.24 & 262 & \multirow[t]{3}{*}{0.50} \\
\hline & & ShMl & 1403 & 0.53 & 435 & 0.57 & 1.08 & 1.23 & 1.80 & 1.10 & 0.32 & 233 & \\
\hline & & ShMl & 1393 & 0.47 & 430 & 0.54 & 1.15 & 2.21 & 2.75 & 0.88 & 0.20 & 470 & \\
\hline & & ShMl & 1387 & 0.39 & & 0.49 & 1.26 & 0.58 & 1.07 & 1.16 & 0.46 & 149 & 0.47 \\
\hline & & ShMl & 1381 & 0.66 & 435 & 0.43 & 0.65 & 3.73 & 4.16 & 2.00 & 0.10 & 565 & \\
\hline & \multirow[t]{2}{*}{ M-2 } & ShMl & 1396 & 0.93 & 429 & 0.16 & 0.17 & 1.27 & 1.43 & 0.72 & 0.11 & 137 & 0.48 \\
\hline & & ShMl & 1426 & 0.84 & 426 & 0.11 & 0.13 & 0.96 & 1.07 & 0.66 & 0.10 & 114 & \\
\hline \multirow[t]{19}{*}{ K } & \multirow[t]{19}{*}{ K-1 } & ShMl & 3523 & 1.91 & 434 & 2.07 & 1.08 & 7.72 & 9.79 & 1.58 & 0.21 & 404 & \\
\hline & & ShMl & 3411 & 1.95 & 434 & 0.58 & 0.30 & 8.39 & 8.97 & 10.11 & 0.06 & 430 & \\
\hline & & Ml & 3353 & 1.92 & 431 & 0.48 & 0.25 & 8.05 & 8.53 & 11.56 & 0.06 & 419 & \\
\hline & & Ml & 3250 & 0.34 & 428 & 0.12 & 0.35 & 0.70 & 0.82 & 1.18 & 0.15 & 206 & \\
\hline & & ShMl & 3238 & 0.35 & 429 & 0.15 & 0.43 & 0.89 & 1.04 & 1.21 & 0.14 & 254 & \\
\hline & & Sh & 3230 & 0.74 & 432 & 0.12 & 0.16 & 1.46 & 1.58 & 1.76 & 0.08 & 197 & \\
\hline & & Sh & 3220 & 0.68 & 432 & 0.12 & 0.18 & 1.46 & 1.58 & 1.50 & 0.07 & 215 & \\
\hline & & Sh & 3210 & 0.75 & 431 & 0.12 & 0.16 & 1.48 & 1.60 & 1.78 & 0.08 & 197 & \\
\hline & & Sh & 3200 & 0.76 & 430 & 0.11 & 0.14 & 1.14 & 1.25 & 1.65 & 0.09 & 150 & \\
\hline & & Sh & 3190 & 0.71 & 430 & 0.15 & 0.21 & 1.19 & 1.34 & 2.04 & 0.12 & 168 & \\
\hline & & Ml & 3175 & 0.68 & 431 & 0.16 & 0.24 & 1.35 & 1.51 & 2.46 & 0.10 & 199 & \\
\hline & & Ml & 3165 & 0.67 & 433 & 0.13 & 0.19 & 1.58 & 1.71 & 1.59 & 0.07 & 236 & \\
\hline & & Ml & 3155 & 0.83 & 435 & 0.26 & 0.31 & 2.34 & 2.60 & 1.71 & 0.10 & 282 & \\
\hline & & Ml & 3145 & 0.48 & 434 & 0.15 & 0.31 & 1.39 & 1.54 & 1.47 & 0.10 & 290 & \\
\hline & & Ml & 3135 & 0.38 & 432 & 0.12 & 0.32 & 1.00 & 1.12 & 1.58 & 0.11 & 263 & \\
\hline & & Ml & 3115 & 0.44 & 433 & 0.12 & 0.27 & 1.33 & 1.45 & 1.53 & 0.08 & 302 & \\
\hline & & Ml & 3105 & 0.35 & 435 & 0.14 & 0.40 & 1.27 & 1.41 & 1.48 & 0.10 & 363 & \\
\hline & & Ml & 3085 & 0.60 & 433 & 0.16 & 0.27 & 1.76 & 1.92 & 1.87 & 0.09 & 293 & \\
\hline & & Ml & 3045 & 0.55 & 435 & 0.17 & 0.31 & 1.46 & 1.63 & 1.50 & 0.10 & 265 & \\
\hline \multirow[t]{5}{*}{$\mathrm{J}$} & \multirow[t]{5}{*}{$\mathrm{J}-1$} & MlSh & 2348 & 0.88 & 435 & 0.23 & 0.26 & 1.07 & 1.30 & 1.32 & 0.18 & 992 & \\
\hline & & MlSh & 2345 & 0.90 & 433 & 0.31 & 0.35 & 1.14 & 1.45 & 1.43 & 0.21 & 947 & \\
\hline & & MlSh & 2340 & 0.78 & 430 & 0.18 & 0.23 & 0.75 & 0.93 & 1.29 & 0.19 & 972 & \\
\hline & & MlSh & 2335 & 0.96 & 433 & 0.29 & 0.30 & 1.31 & 1.60 & 1.53 & 0.18 & 986 & \\
\hline & & MlSh & 2140 & 0.39 & 426 & 0.32 & 0.82 & 0.60 & 0.92 & 1.24 & 0.35 & 786 & 0.55 \\
\hline \multirow[t]{12}{*}{ I } & \multirow[t]{3}{*}{$\mathrm{I}-2$} & ShMl & 1891 & 1.62 & 430.0 & 0.41 & 0.25 & 3.46 & 3.87 & & 0.11 & 214 & \\
\hline & & ShMl & 1942 & 0.62 & 434.0 & 0.21 & 0.34 & 0.58 & 0.79 & & 0.27 & 94 & \\
\hline & & M1 & 1980 & 0.82 & 432.0 & 0.21 & 0.26 & 1.31 & 1.52 & & 0.14 & 160 & \\
\hline & \multirow[t]{9}{*}{ I-1 } & Sh & 2696 & 0.80 & 426 & 0.12 & 0.15 & 0.70 & 0.82 & 1.50 & 0.14 & 88 & \multirow[t]{9}{*}{0.56} \\
\hline & & ShMl & 2660 & 1.83 & 424 & 0.77 & 0.42 & 6.92 & 7.69 & 3.67 & 0.10 & 378 & \\
\hline & & ShMl & 2650 & 1.81 & 403 & 0.54 & 0.30 & 3.55 & 4.09 & 8.21 & 0.13 & 196 & \\
\hline & & Ml & 2640 & 0.68 & 413 & 0.35 & 0.51 & 1.43 & 1.78 & 3.51 & 0.20 & 210 & \\
\hline & & ShMl & 2625 & 0.50 & 432 & 0.12 & 0.24 & 1.38 & 1.50 & 2.13 & 0.08 & 276 & \\
\hline & & ShMl & 2605 & 0.62 & 433 & 0.12 & 0.19 & 1.97 & 2.09 & 2.16 & 0.06 & 318 & \\
\hline & & ShMl & 2595 & 0.47 & 433 & 0.11 & 0.23 & 1.33 & 1.44 & 1.94 & 0.08 & 283 & \\
\hline & & LimMl & 2585 & 0.63 & 430 & 0.10 & 0.16 & 1.06 & 1.16 & 1.85 & 0.08 & 168 & \\
\hline & & Ml & 2575 & 0.50 & 432 & 0.09 & 0.18 & 1.06 & 1.15 & 2.00 & 0.08 & 212 & \\
\hline
\end{tabular}


Table 2 continued

\begin{tabular}{|c|c|c|c|c|c|c|c|c|c|c|c|c|c|}
\hline Field & Well & Lith. $^{\mathrm{a}}$ & $\mathrm{MD}, \mathrm{m}$ & TOC & $T_{\max }$ & $S_{1}$ & $S_{1} / \mathrm{TOC}$ & $S_{2}$ & $S_{1}+S_{2}$ & $S_{3}$ & PI & HI & $R_{\mathrm{o}}, \%$ \\
\hline & & Ml & 2565 & 0.51 & 434 & 0.10 & 0.20 & 0.96 & 1.06 & 1.86 & 0.10 & 188 & \\
\hline & & Ml & 2555 & 0.50 & 433 & 0.24 & 0.48 & 1.22 & 1.46 & 1.93 & 0.17 & 244 & \\
\hline & & Ml & 2535 & 0.77 & 431 & 0.24 & 0.31 & 2.43 & 2.67 & 2.10 & 0.09 & 316 & \\
\hline & & Ml & 2525 & 0.70 & 432 & 0.20 & 0.29 & 1.98 & 2.18 & 1.98 & 0.09 & 283 & \\
\hline & & Ml & 2515 & 0.69 & 431 & 0.20 & 0.29 & 1.88 & 2.08 & 1.98 & 0.09 & 272 & \\
\hline & & Ml & 2503 & 0.54 & 434 & 0.15 & 0.28 & 1.56 & 1.71 & 1.96 & 0.08 & 289 & \\
\hline & & Ml & 2495 & 0.81 & 432 & 0.18 & 0.22 & 1.74 & 1.92 & 2.58 & 0.09 & 215 & \\
\hline & & Ml & 2475 & 0.98 & 431 & 0.36 & 0.37 & 2.25 & 2.61 & 2.86 & 0.14 & 230 & \\
\hline & & Ml & 2445 & 1.17 & 430 & 0.50 & 0.43 & 3.32 & 3.82 & 3.81 & 0.13 & 284 & \\
\hline & & Ml & 2413 & 0.80 & 430 & 0.26 & 0.33 & 2.80 & 3.06 & 1.75 & 0.08 & 350 & \\
\hline & & Ml & 2405 & 0.84 & 432 & 0.22 & 0.26 & 2.71 & 2.93 & 1.82 & 0.08 & 323 & \\
\hline & & Ml & 2395 & 0.90 & 431 & 0.14 & 0.16 & 3.00 & 3.14 & 1.77 & 0.04 & 333 & \\
\hline & & Ml & 2385 & 0.99 & 431 & 0.17 & 0.17 & 3.13 & 3.30 & 1.79 & 0.05 & 316 & \\
\hline & & Ml & 2375 & 1.05 & 430 & 0.22 & 0.21 & 3.67 & 3.89 & 1.96 & 0.06 & 350 & \\
\hline \multirow[t]{3}{*}{$\mathrm{O}$} & O-2 & Ml & 2985 & 0.34 & & 0.23 & 0.68 & 0.62 & 0.85 & & 0.27 & 182 & 0.65 \\
\hline & & Ml & 3165 & 0.63 & 433 & 0.48 & 0.76 & 0.92 & 1.40 & & 0.34 & 146 & 0.70 \\
\hline & & Ml & 3362 & 1.28 & 439 & 0.75 & 0.59 & 2.06 & 2.81 & & 0.27 & 161 & 0.75 \\
\hline
\end{tabular}

The units of the Rock-Eval pyrolysis parameters and indices: TOC wt $\%, S_{1} \mathrm{mg} \mathrm{HC/g}$ rock, $S_{2} \mathrm{mg} \mathrm{HC} / \mathrm{g}$ rock, $S_{1}+S_{2} \mathrm{mg} \mathrm{HC} / \mathrm{g}$ rock, $\mathrm{S}_{3} \mathrm{mg} \mathrm{CO} / \mathrm{g}$ rock, $T_{\max }{ }^{\circ} \mathrm{C}, \mathrm{HI} \mathrm{mg} \mathrm{HC} / \mathrm{g}$ TOC, OI $\mathrm{mg} \mathrm{CO} / \mathrm{g}_{2}$ TOC

a $\mathrm{ShMl}$ is shaly marl, $\mathrm{Ml}$ is marl, Sh is shale, and MlSh is marly shale

Table 3 Rock-Eval and vitrinite reflectance data for the Ahmadi samples

\begin{tabular}{|c|c|c|c|c|c|c|c|c|c|c|c|c|c|}
\hline Field & Well & Lith. $^{\mathrm{a}}$ & $\mathrm{MD}, \mathrm{m}$ & TOC & $T_{\max }$ & $S_{1}$ & $S_{1} / \mathrm{TOC}$ & $S_{2}$ & $S_{1}+S_{2}$ & $S_{3}$ & PI & HI & $R_{\mathrm{o}}, \%$ \\
\hline \multirow[t]{2}{*}{$\mathrm{J}$} & \multirow[t]{2}{*}{$\mathrm{J}-1$} & Ml & 2561 & 1.60 & 440 & 1.83 & 1.14 & 4.25 & 6.08 & 1.38 & 0.30 & 842 & \\
\hline & & Ml & 2561 & 0.52 & 432 & 0.37 & 0.71 & 0.61 & 0.98 & 1.24 & 0.38 & 750 & 0.67 \\
\hline \multirow[t]{2}{*}{ I } & \multirow[t]{2}{*}{$\mathrm{I}-2$} & Ml & 2123 & 1.37 & 422 & 0.41 & 0.30 & 7.98 & 8.39 & 0.53 & 0.05 & 582 & \\
\hline & & Ml & 2138 & 2.68 & 424 & 1.19 & 0.44 & 16.94 & 18.13 & 0.70 & 0.07 & 632 & \\
\hline \multirow[t]{2}{*}{ D } & \multirow[t]{2}{*}{ D-1 } & Ml & 1924 & 0.42 & & 0.08 & 0.19 & 0.36 & 0.44 & 2.01 & 0.19 & 86 & 0.39 \\
\hline & & Ml & 1846 & 0.30 & & 0.11 & 0.37 & 0.21 & 0.32 & 1.58 & 0.34 & 70 & 0.36 \\
\hline \multirow[t]{4}{*}{$\mathrm{C}$} & \multirow[t]{4}{*}{ C-1 } & MlSh & 1930 & 1.25 & 428 & 0.78 & 0.62 & 1.66 & 2.44 & 2.15 & 0.32 & 133 & 0.50 \\
\hline & & MlSh & 1910 & 1.25 & 431 & 0.62 & 0.50 & 1.67 & 2.29 & 1.93 & 0.27 & 134 & \\
\hline & & MlSh & 1886 & 1.08 & 432 & 0.89 & 0.82 & 1.75 & 2.64 & 1.48 & 0.34 & 162 & \\
\hline & & MlSh & 1902 & 1.02 & 426 & 0.90 & 0.88 & 1.73 & 2.63 & 2.23 & 0.34 & 170 & \\
\hline \multirow[t]{6}{*}{ B } & \multirow[t]{6}{*}{ B-1 } & ShMl & 2520 & 0.45 & 432 & 0.21 & 0.47 & 0.64 & 0.85 & 0.85 & 0.25 & 143 & \\
\hline & & ShMl & 2510 & 0.51 & 433 & 0.18 & 0.35 & 0.70 & 0.88 & 0.87 & 0.20 & 138 & \\
\hline & & MlSh & 2490 & 0.98 & 435 & 0.16 & 0.16 & 1.11 & 1.27 & 1.21 & 0.13 & 113 & \\
\hline & & MlSh & 2450 & 1.34 & 425 & 0.35 & 0.26 & 5.42 & 5.77 & 1.16 & 0.06 & 404 & \\
\hline & & Ml & 2420 & 3.86 & 421 & 0.96 & 0.25 & 14.16 & 15.12 & 2.73 & 0.06 & 367 & \\
\hline & & Ml & 2380 & 0.38 & 435 & 0.16 & 0.42 & 0.52 & 0.68 & 0.73 & 0.24 & 138 & 0.57 \\
\hline $\mathrm{O}$ & $\mathrm{O}-2$ & Ml & 3453 & 0.44 & 442 & 0.46 & 1.05 & 1.94 & 2.40 & 0.82 & 0.19 & 441 & 0.80 \\
\hline M & M-2 & MlSh & 1555 & 0.33 & 419 & 0.05 & 0.15 & 0.11 & 0.16 & 1.09 & 0.33 & 33 & \\
\hline
\end{tabular}

The units of the Rock-Eval pyrolysis parameters and indices: TOC wt $\%, S_{1} \mathrm{mg} \mathrm{HC} / \mathrm{g}$ rock, $S_{2} \mathrm{mg} \mathrm{HC} / \mathrm{g}$ rock, $S_{1}+S_{2} \mathrm{mg} \mathrm{HC} / \mathrm{g}$ rock, $\mathrm{S}_{3} \mathrm{mg} \mathrm{CO} / \mathrm{g}$ rock, $T_{\max }{ }^{\circ} \mathrm{C}$, $\mathrm{HI} \mathrm{mg} \mathrm{HC} / \mathrm{g}$ TOC, OI $\mathrm{mg} \mathrm{CO}_{2} / \mathrm{g}$ TOC

a $\mathrm{Ml}$ is marl, MlSh is marly shale, and ShMl is shaly marl 
Table 4 Rock-Eval and vitrinite reflectance data for the Kazhdumi samples

\begin{tabular}{|c|c|c|c|c|c|c|c|c|c|c|c|c|c|}
\hline Field & Well & Lith. $^{\mathrm{a}}$ & $\mathrm{MD}, \mathrm{m}$ & TOC & $T_{\max }$ & $S_{1}$ & $S_{1} / \mathrm{TOC}$ & $S_{2}$ & $S_{1}+S_{2}$ & $S_{3}$ & PI & $\mathrm{HI}$ & $R_{\mathrm{o}}, \%$ \\
\hline \multirow[t]{2}{*}{ M } & M-1 & ShMl & 1555 & 0.34 & 431 & 0.46 & 1.35 & 0.51 & 0.97 & 1.11 & 0.48 & 150 & \\
\hline & M-2 & ShMl & 1588 & 0.30 & 410 & 0.05 & 0.17 & 0.10 & 0.15 & 1.14 & 0.35 & 33 & \\
\hline $\mathrm{F}$ & F-1 & Sh & 1468 & 0.19 & 416 & 0.24 & 1.26 & 0.16 & 0.40 & 0.74 & 0.60 & 84 & 0.50 \\
\hline $\mathrm{D}$ & D-1 & Ml & 2026 & 0.39 & 425 & 0.19 & 0.49 & 0.71 & 0.90 & 1.77 & 0.21 & 182 & 0.41 \\
\hline \multirow[t]{18}{*}{$\mathrm{C}$} & $\mathrm{C}-1$ & LimMl & 2108 & 0.72 & 422 & 0.75 & 1.04 & 1.00 & 1.75 & 2.33 & 0.43 & 139 & 0.47 \\
\hline & & Sh & 2091 & 0.86 & 423 & 0.85 & 0.99 & 1.23 & 2.08 & 2.89 & 0.41 & 143 & \\
\hline & & Sh & 2086 & 1.13 & 428 & 0.99 & 0.88 & 2.04 & 3.03 & 2.94 & 0.33 & 181 & \\
\hline & & Sh & 2070 & 1.68 & 428 & 1.62 & 0.96 & 3.47 & 5.09 & 1.61 & 0.32 & 207 & \\
\hline & & Sh & 2060 & 2.83 & 429 & 2.24 & 0.79 & 6.62 & 8.86 & 1.41 & 0.25 & 234 & 0.54 \\
\hline & & $\mathrm{MlSh}$ & 2033 & 2.79 & 423 & 1.48 & 0.53 & 6.45 & 7.93 & 2.47 & 0.19 & 231 & 0.47 \\
\hline & & Sh & 2029 & 2.64 & 426 & 1.27 & 0.48 & 4.44 & 5.71 & 2.84 & 0.22 & 168 & \\
\hline & & Sh & 2022 & 2.47 & 426 & 1.29 & 0.52 & 5.08 & 6.37 & 2.70 & 0.20 & 206 & 0.51 \\
\hline & & Sh & 2014 & 2.19 & 426 & 1.26 & 0.58 & 4.86 & 6.12 & 2.69 & 0.21 & 222 & \\
\hline & & Sh & 2006 & 1.99 & 430 & 1.19 & 0.60 & 3.84 & 5.03 & 2.34 & 0.24 & 193 & 0.56 \\
\hline & & Sh & 1998 & 1.94 & 430 & 1.00 & 0.52 & 3.49 & 4.49 & 2.94 & 0.22 & 180 & \\
\hline & & Sh & 1991 & 2.13 & 429 & 1.20 & 0.56 & 3.79 & 4.99 & 2.77 & 0.24 & 178 & 0.53 \\
\hline & & MlSh & 1987 & 1.33 & 430 & 0.86 & 0.65 & 2.24 & 3.10 & 2.14 & 0.28 & 168 & 0.61 \\
\hline & & $\mathrm{MlSh}$ & 1979 & 1.01 & 431 & 0.79 & 0.78 & 1.71 & 2.50 & 1.51 & 0.32 & 169 & 0.64 \\
\hline & & MlSh & 1970 & 0.70 & 430 & 0.75 & 1.07 & 1.05 & 1.80 & 1.61 & 0.42 & 150 & \\
\hline & & Ml & 1967 & 0.51 & 429 & 0.54 & 1.06 & 0.72 & 1.26 & 1.78 & 0.43 & 141 & \\
\hline & & Ml & 1952 & 0.34 & 425 & 0.57 & 1.68 & 0.62 & 1.19 & 1.65 & 0.48 & 182 & \\
\hline & & Ml & 1940 & 1.16 & 428 & 0.72 & 0.62 & 1.54 & 2.26 & 2.00 & 0.32 & 133 & \\
\hline \multirow[t]{22}{*}{ B } & B-1 & Sh & 2970 & 1.02 & 437 & 0.14 & 0.14 & 1.08 & 1.22 & 0.96 & 0.11 & 106 & \\
\hline & & Sh & 2960 & 1.17 & 435 & 0.20 & 0.17 & 1.76 & 1.96 & 1.26 & 0.10 & 151 & 0.69 \\
\hline & & Sh & 2950 & 1.34 & 437 & 0.29 & 0.22 & 2.09 & 2.38 & 1.32 & 0.12 & 157 & \\
\hline & & Sh & 2930 & 1.66 & 438 & 0.31 & 0.19 & 2.21 & 2.52 & 1.95 & 0.12 & 133 & 0.68 \\
\hline & & Sh & 2920 & 1.38 & 440 & 0.18 & 0.13 & 1.89 & 2.07 & 1.71 & 0.09 & 137 & \\
\hline & & Sh & 2910 & 0.95 & 440 & 0.18 & 0.19 & 1.32 & 1.50 & 0.92 & 0.12 & 138 & 0.66 \\
\hline & & Sh & 2900 & 0.80 & 461 & 0.10 & 0.13 & 0.50 & 0.60 & 1.44 & 0.17 & 63 & \\
\hline & & MlSh & 2890 & 1.22 & 434 & 0.38 & 0.31 & 2.25 & 2.63 & 1.00 & 0.14 & 184 & 0.66 \\
\hline & & MlSh & 2880 & 1.43 & 427 & 0.94 & 0.66 & 3.67 & 4.61 & 0.83 & 0.20 & 257 & \\
\hline & & MlSh & 2870 & 1.39 & 425 & 1.32 & 0.95 & 4.62 & 5.94 & 0.83 & 0.22 & 332 & 0.65 \\
\hline & & MlSh & 2860 & 0.83 & 435 & 0.21 & 0.25 & 1.34 & 1.55 & 1.06 & 0.14 & 161 & \\
\hline & & MlSh & 2850 & 0.78 & 434 & 0.28 & 0.36 & 1.27 & 1.55 & 1.01 & 0.18 & 162 & \\
\hline & & MlSh & 2840 & 0.96 & 433 & 0.24 & 0.25 & 1.74 & 1.98 & 1.15 & 0.12 & 181 & 0.64 \\
\hline & & MlSh & 2830 & 0.83 & 437 & 0.25 & 0.30 & 1.12 & 1.37 & 1.02 & 0.18 & 135 & \\
\hline & & MlSh & 2820 & 0.93 & 427 & 0.71 & 0.76 & 3.10 & 3.81 & 0.90 & 0.19 & 333 & 0.62 \\
\hline & & MlSh & 2800 & 0.74 & 436 & 0.15 & 0.20 & 0.44 & 0.59 & 1.48 & 0.25 & 59 & \\
\hline & & MlSh & 2790 & 0.82 & 436 & 0.16 & 0.19 & 0.90 & 1.06 & 1.27 & 0.15 & 109 & \\
\hline & & Sh & 2780 & 1.03 & 436 & 0.20 & 0.19 & 1.30 & 1.50 & 1.14 & 0.13 & 126 & 0.62 \\
\hline & & Sh & 2770 & 1.02 & 437 & 0.18 & 0.18 & 1.10 & 1.28 & 1.16 & 0.14 & 107 & \\
\hline & & MlSh & 2760 & 1.31 & 434 & 0.40 & 0.31 & 2.43 & 2.83 & 1.59 & 0.14 & 185 & 0.61 \\
\hline & & MlSh & 2750 & 0.78 & 436 & 0.21 & 0.27 & 0.96 & 1.17 & 1.07 & 0.18 & 124 & \\
\hline & & ShMl & 2740 & 0.72 & 429 & 0.18 & 0.25 & 1.28 & 1.46 & 0.85 & 0.12 & 178 & 0.60 \\
\hline \multirow[t]{3}{*}{$\mathrm{O}$} & $\mathrm{O}-2$ & ShMl & 3548 & 0.57 & 436 & 0.32 & 0.56 & 1.89 & 2.21 & 2.11 & 0.14 & 332 & 0.80 \\
\hline & & ShMl & 3595 & 0.70 & 464 & 0.18 & 0.26 & 3.81 & 3.99 & 1.43 & 0.05 & 544 & 0.81 \\
\hline & & ShMl & 3603 & 0.54 & 463 & 0.17 & 0.31 & 3.21 & 3.38 & 0.91 & 0.05 & 594 & 0.82 \\
\hline
\end{tabular}


Table 4 continued

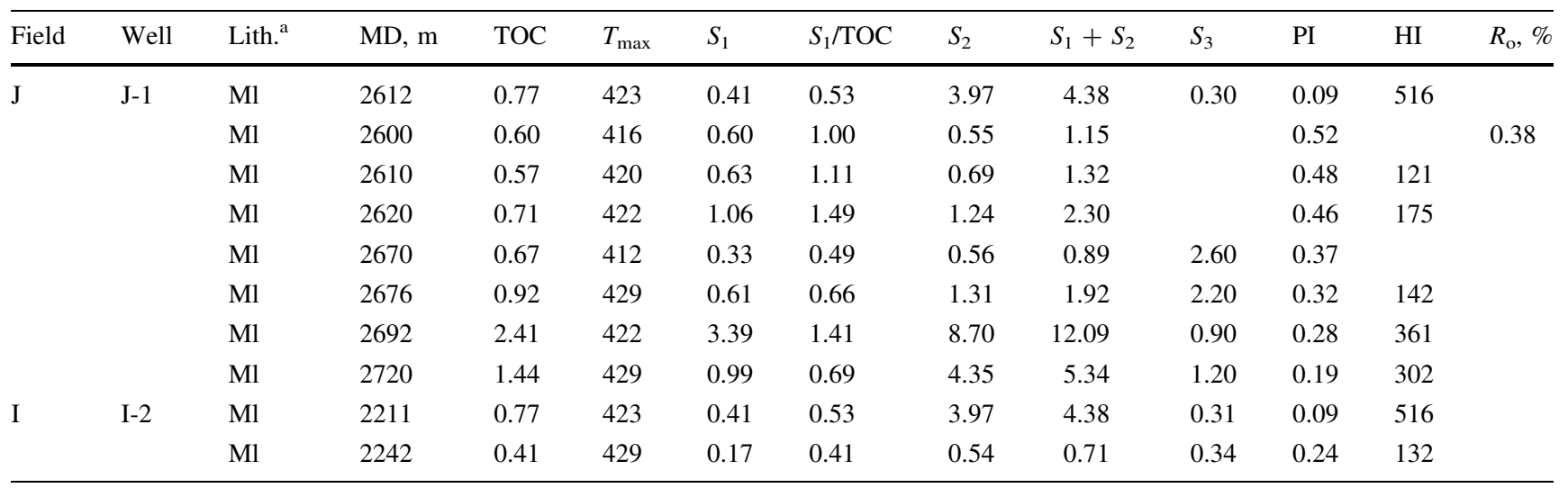

The units of the Rock-Eval pyrolysis parameters and indices: TOC wt $\%, S_{1} \mathrm{mg} \mathrm{HC} / \mathrm{g}$ rock, $S_{2} \mathrm{mg} \mathrm{HC} / \mathrm{g}$ rock, $S_{1}+S_{2} \mathrm{mg} \mathrm{HC} / \mathrm{g}$ rock, $\mathrm{S}_{3} \mathrm{mg} \mathrm{CO} / \mathrm{g}$ rock, $T_{\max }{ }^{\circ} \mathrm{C}$, $\mathrm{HI} \mathrm{mg} \mathrm{HC} / \mathrm{g}$ TOC, OI $\mathrm{mg} \mathrm{CO}_{2} / \mathrm{g}$ TOC

a $\mathrm{ShMl}$ is shaly marl, $\mathrm{Ml}$ is marl, $\mathrm{Sh}$ is shale, MlSh is marly shale, and LiMl is limy marl

in the organic petrography laboratory of Research Institute of Petroleum Industry (RIPI) in Iran. A sapphire glass standard with a $0.589 \%$ reflectance value was used for calibration. The measurements were performed under reflected light at a wavelength of $546 \mathrm{~nm}$ with an oil immersion objective with $\times 125$ magnifications. At least, 50 readings were performed for each sample.

Basin modeling is a useful method for investigating the burial and thermal evolutions of sedimentary basins. In this study, six selected wells were modeled using PetroMod-1D modeling software (version 11, Schlumberger). The selected wells include B-1 (within the Field B, in the western part of the study area), C-1 (within the Field C, in the western part of the study area), D-1 (within the Field D, in the Central Persian Gulf), F-1 (within the Field F, in the Central Persian Gulf), J-1 (within the Field J, in the eastern part of the study area), and P-1 (within the Field P, in the eastern part of the study area). The location of the fields is shown in Fig. 1. Important 1D model input parameters involve the burial depths, thickness of the strata, erosion thickness and time, lithologies, kerogen types and kinetics, and further geochemical parameters such as the initial $\%$ TOC and HI (Table 6). The lithological information was inferred from unpublished well log data from National Iranian Oil Company (NIOC). The absolute ages were obtained from the timescale and regional chronostratigraphic subdivisions of Gradstein et al. (2004). The thermal evolution is modeled based on boundary conditions including the sediment water interface temperature (SWIT, in ${ }^{\circ} \mathrm{C}$ ), paleo-water depth (PWD, in meter), and heat flow (in $\mathrm{mW} / \mathrm{m}^{2}$ ).

The upper boundary condition for calculating the temperature development in a sedimentary basin is the SWIT (Yalçin et al. 1997). The PetroMod-1D software estimates the SWIT values through time based on the approach developed by Wygrala (1989). This estimation is based on the paleogeographical position of the area through geological time, variations in the mean surface paleo temperatures versus latitude and geological time; and water depth during the time of deposition (Yalçin et al. 1997). The SWIT calculations of this study were based on the paleo-latitude of the Northern Arabian plate.

The PWD values are required to calculate the SWIT. The PWD is dependent on combination of tectonic subsidence and changes in global sea levels. The depositional environment of each formation gives information about the PWD. A PWD of $0 \mathrm{~m}$ was considered for erosional events or phases of non-deposition and values of $20 \mathrm{~m}$ were applied for times of carbonate deposition. Negative PWD values were not used in this study.

The heat flow is the lower boundary condition of heat transfer into a sedimentary basin (Yalçin et al. 1997). It is an important input parameter in basin modeling and usually difficult to define for the geological past. Therefore, thermal history models are commonly calibrated against maturity and temperature profiles. In this study, bottomhole temperature and vitrinite reflectance data were used for the temperature and maturity calibrations, respectively. Recently, heat flow values in the range of $60-68 \mathrm{~mW} / \mathrm{m}^{2}$ were shown to be in accordance with the vitrinite reflectance measurements in the central Persian Gulf (Mohsenian et al. 2014). The easy $\% R_{\mathrm{o}}$ kinetic model of Sweeney and Burnham (1990) was applied to calculate the thermal maturity levels of the studied formations. Petroleum generation stages were calculated assuming mainly Type II kerogen and using a reaction kinetic dataset based on Burnham (1989). 
Table 5 Isoprenoids ratios measured for the studied samples

\begin{tabular}{|c|c|c|c|c|c|c|c|}
\hline Field & Well & Formation & Lith. & Depth, $m$ & $\mathrm{Pr} / \mathrm{Ph}$ & $\mathrm{Pr} / n-\mathrm{C}_{17}$ & $\mathrm{Ph} / n-\mathrm{C}_{18}$ \\
\hline \multirow[t]{2}{*}{ I } & $\mathrm{I}-1$ & Gurpi & Ml & 2445 & 0.42 & 0.41 & 0.51 \\
\hline & $\mathrm{I}-1$ & Gurpi & Ml & 2395 & 0.28 & 0.51 & 0.60 \\
\hline \multirow[t]{2}{*}{$\mathrm{L}$} & L-1 & Gurpi & MlSh & 2857 & 0.36 & 0.55 & 0.77 \\
\hline & L-1 & Gurpi & ShMl & 2767 & 0.31 & 0.51 & 0.73 \\
\hline $\mathrm{H}$ & $\mathrm{H}-1$ & Gurpi & $\mathrm{Sh}$ & 2199 & 0.12 & 0.44 & 0.69 \\
\hline $\mathrm{E}$ & E-1 & Kazhdumi & $\mathrm{Sh}$ & 1631 & 0.20 & 0.56 & 0.94 \\
\hline $\mathrm{G}$ & G-1 & Kazhdumi & ShMl & 1504 & 0.30 & 0.36 & 0.63 \\
\hline $\mathrm{N}$ & $\mathrm{N}-1$ & Kazhdumi & Sh & 2000 & 0.56 & 0.84 & 1.42 \\
\hline A & A-1 & Kazhdumi & $\mathrm{Sh}$ & 3070 & 0.41 & 0.44 & 0.79 \\
\hline \multirow[t]{3}{*}{$\mathrm{C}$} & $\mathrm{C}-1$ & Kazhdumi & Sh-MlSh & 2048 & 0.42 & 0.62 & 0.94 \\
\hline & $\mathrm{C}-1$ & Kazhdumi & Sh & 1999 & 0.59 & 0.53 & 0.94 \\
\hline & $\mathrm{C}-1$ & Kazhdumi & MlSh & 1982 & 0.34 & 0.58 & 0.97 \\
\hline \multirow[t]{4}{*}{$\mathrm{L}$} & L-1 & Pabdeh & Ml & 2238 & 0.13 & 0.23 & 0.61 \\
\hline & L-1 & Pabdeh & Ml & 2137 & 0.39 & 0.55 & 0.81 \\
\hline & L-1 & Pabdeh & Ml & 1968 & 0.28 & 0.55 & 0.82 \\
\hline & L-1 & Ahmadi Mbr. & MlSh & 3148 & 0.27 & 0.38 & 0.59 \\
\hline $\mathrm{H}$ & $\mathrm{H}-1$ & Ahmadi Mbr. & $\mathrm{Sh}$ & 2574 & 0.12 & 0.37 & 0.80 \\
\hline $\mathrm{G}$ & G-1 & Ahmadi Mbr. & Ml & 1412 & 0.11 & 0.54 & 0.60 \\
\hline
\end{tabular}

Table 6 Examples of input data for burial, thermal maturity, and hydrocarbon generation modeling in well P-1

\begin{tabular}{|c|c|c|c|c|c|c|c|c|c|}
\hline Formation & $\begin{array}{l}\text { Top, } \\
\text { m }\end{array}$ & $\begin{array}{l}\text { Base, } \\
\mathrm{m}\end{array}$ & $\begin{array}{l}\text { Thickness, } \\
\mathrm{m}\end{array}$ & $\begin{array}{l}\text { Eroded, } \\
\mathrm{m}\end{array}$ & $\begin{array}{l}\text { Deposition } \\
\text { from, Ma }\end{array}$ & $\begin{array}{l}\text { Deposition } \\
\text { to, Ma }\end{array}$ & $\begin{array}{l}\text { Eroded } \\
\text { from, Ma }\end{array}$ & $\begin{array}{l}\text { Eroded } \\
\text { to, } \mathrm{Ma}\end{array}$ & Lithology \\
\hline Bakhtiyari & 70 & 100 & 30 & 0 & 10.87 & 1.5 & 1.5 & 0 & Sandstone and marl \\
\hline Upper Fars & 100 & 914 & 814 & & 13.3 & 10.87 & & & Marl and Lime-Marly \\
\hline Lower Fars & 914 & 1200 & 286 & & 20 & 13.3 & & & Evaporite \\
\hline Asmari & 1200 & 1514 & 314 & & 23 & 20 & & & Limestone \\
\hline Pabdeh & 1514 & 2972 & 1458 & 200 & 59.33 & 35 & 35 & 23 & Limestone and marl \\
\hline Gurpi & 2972 & 3695 & 723 & 200 & 81.01 & 64.02 & 64.02 & 59.33 & Limestone and marl \\
\hline Ilam & 3695 & 3715 & 20 & & 83.43 & 81.01 & & & Limestone \\
\hline Laffan & 3715 & 3716 & 2 & & 88.29 & 83.43 & & & Shale \\
\hline Sarvak-Mishrif & 3716 & 3799 & 83 & 50 & 92.97 & 90.71 & 90.71 & 88.29 & Limestone \\
\hline Sarvak-Ahmadi & 3799 & 3827 & 28 & & 98 & 95 & & & Limestone and marl \\
\hline Sarvak-Mauddud & 3827 & 3912 & 85 & & 102.77 & 98 & & & Limestone \\
\hline Kazhdumi & 3912 & 4045 & 133 & & 115 & 102.77 & & & Limestone and marl \\
\hline
\end{tabular}

\section{Results and discussions}

\subsection{Rock-Eval data}

Tables 1, 2, 3 and 4 and Fig. 5 show the results of the Rock-Eval pyrolysis in the studied wells. The cross-plot of $S_{1}$ versus TOC discriminates the nonindigenous and indigenous nature of the hydrocarbons present in the source rock samples (Hunt 1996). A migration index ( $S_{1} /$ TOC) greater than 1.5 reveals that migrated hydrocarbons affected the samples, whereas an index less than 1.5 points to an indigenous nature for the hydrocarbons. All of the studied samples have migration indices lower than 1.5 , indicating that the analyzed samples were not polluted by migrated hydrocarbons (Fig. 6; Tables 1, 2, 3, 4).

The TOC content and genetic potential (summation of $S_{1}$ and $S_{2}$ peaks of Rock-Eval pyrolysis) provide important information about hydrocarbon generation potential of the source rocks. The TOC contents of the studied samples are in the range of $0.2-3.86 \mathrm{wt} \%$ with values generally lower 

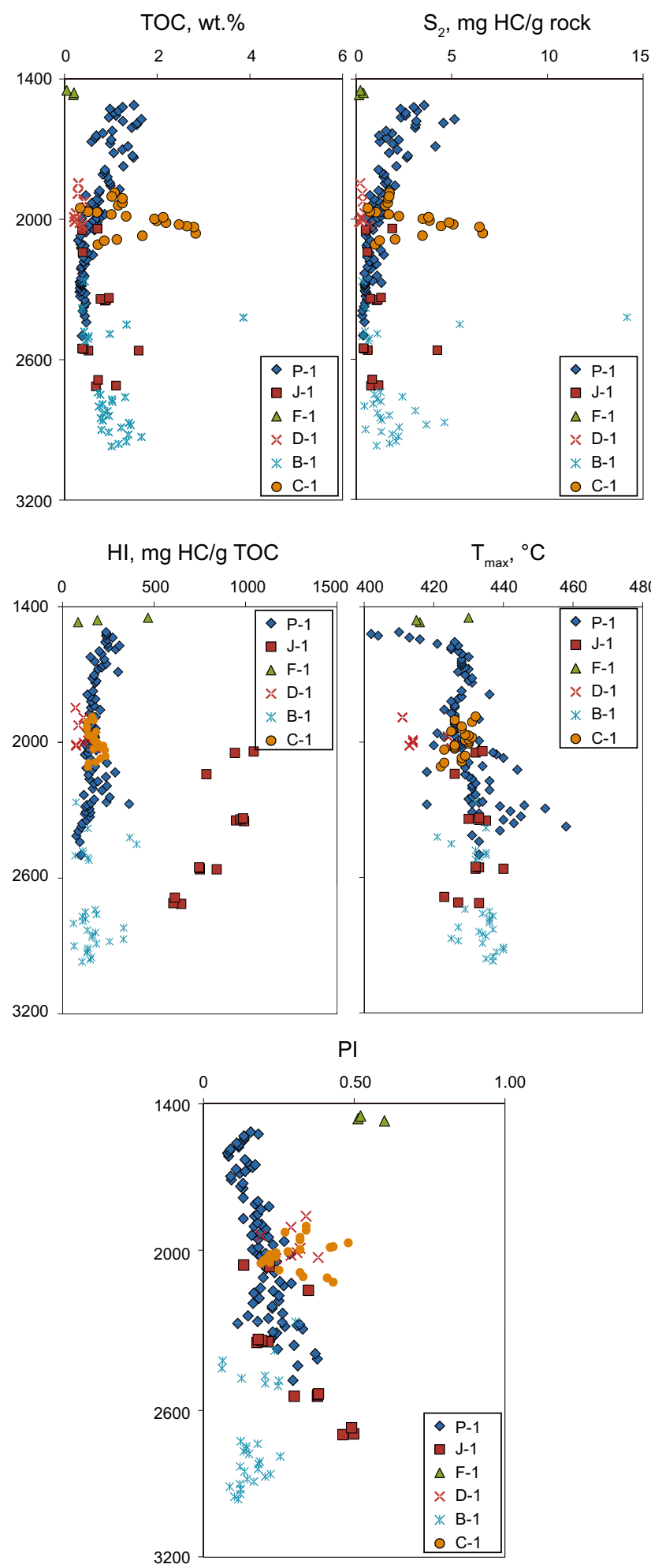

Fig. 5 Geochemical logs showing Rock-Eval data in the studied wells

than $2 \mathrm{wt} \%$ (Tables 1, 2, 3, 4). The genetic potential varies between 0.2 and $18.13 \mathrm{mg} \mathrm{HC} / \mathrm{g}$ rock with values mostly lower than $6 \mathrm{mg} \mathrm{HC/g}$ rock (Tables $1,2,3,4)$. The $S_{2}$

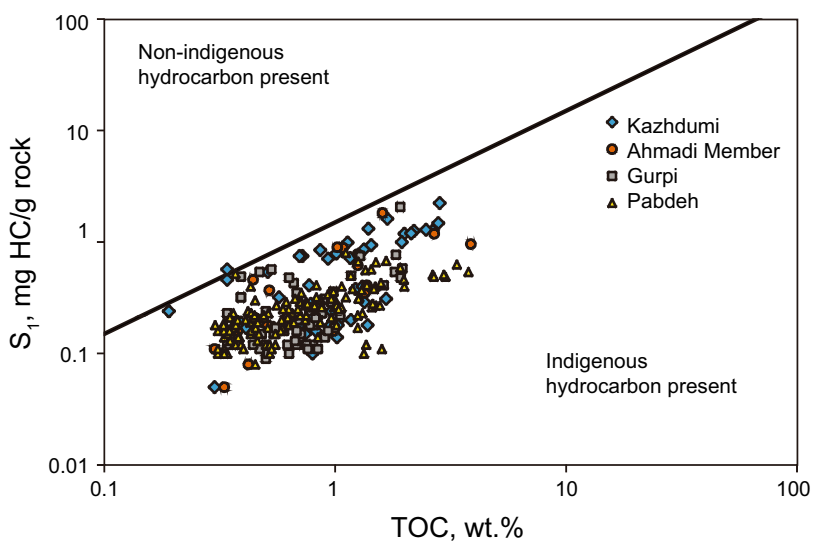

Fig. 6 Plot of $S_{1}$ versus TOC for distinguishing between indigenous and nonindigenous hydrocarbons present in the samples in the Kazhdumi (right), Gurpi (middle), and Pabdeh (left) formations (after Hunt 1996). The inclined line represents $S_{1} /$ TOC $=1.5$

values measured for the studied samples are in the range of $0.5-17 \mathrm{mg} \mathrm{HC} / \mathrm{g}$ rock with average of $2.5 \mathrm{mg} \mathrm{HC} / \mathrm{g}$ rock. The cross-plots of genetic potential versus TOC (Fig. 7a) and $S_{2}$ versus TOC (Fig. 7b) indicate that the studied samples can be generally regarded as having a fair generative potential of hydrocarbon. HI values are in the range of 33-991 mg HC/g TOC (Tables 1, 2, 3, 4), and fair petroleum generation potential of the studied samples is also evident by cross-plot of HI versus TOC (Fig. 7c). Generally, the studied samples show lower TOC, genetic potential, and HI values in the central wells of the Persian Gulf (Tables 1, 2, 3, 4). This is in agreement with increasing the thickness of the studied source rocks from the Central Persian Gulf toward the eastern and western parts (Fig. 4). The occurrence of uplift in the Central Persian Gulf due to the presence of the Qatar Arch resulted in relatively poor preservation of organic matter in this part of the Persian Gulf compared to the adjacent areas.

The type of organic matter present in the source rocks can be evaluated based on the modified Van Krevelen diagram of $\mathrm{HI}$ versus $T_{\max }$. The analyzed samples mainly plotted in the zone of mixed Type II-III kerogens and Type III kerogen of this diagram (Fig. 8a). Moreover, in the $S_{2}$ TOC plot (Fig. 8b), most of the samples fall in the zone of mixed Types II-III kerogens grading to Type III. The local source rock evaluation study of Ghasemi-Nejad et al. (2009) in the South Pars Field also reveals this type of kerogen for the Kazhdumi Formation. This type of kerogen may originate from mixtures of terrigenous and marine organic matter with varying oil and gas generation potential or may also originally be a marine Type II organic matter which has partially been oxidized during deposition. As shown in the following section, the latter interpretation is the more likely. 
(a)

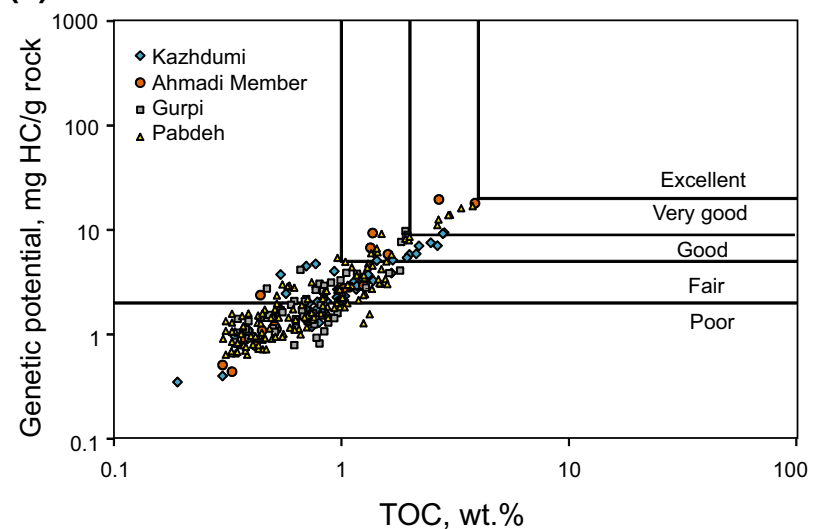

(b)

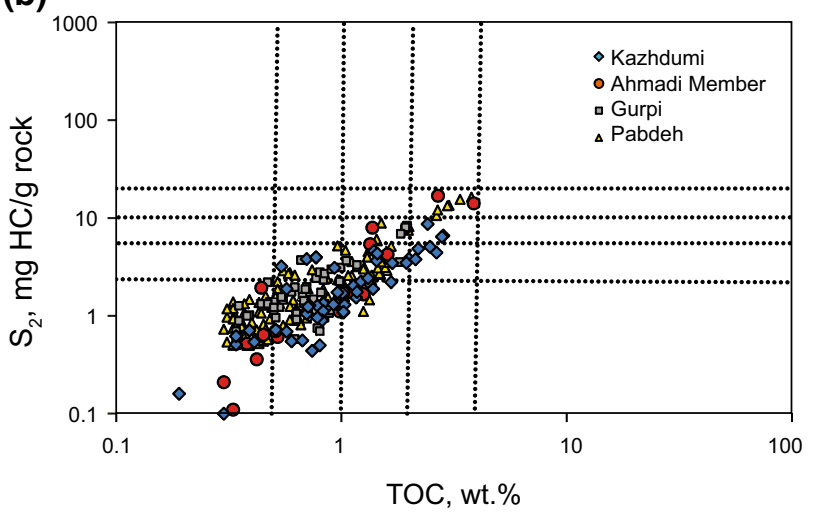

(c)

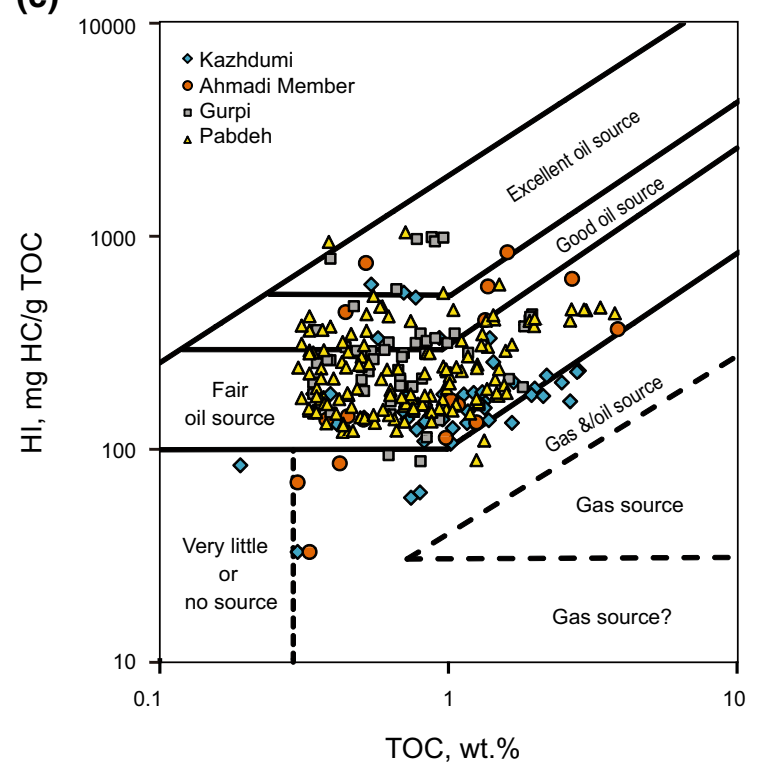

Fig. 7 Plots of a the genetic potential versus TOC, b $S_{2}$ versus TOC, and $\mathbf{c}$ HI versus TOC diagram showing source rock quality of the studied samples

\subsection{Normal alkane and isoprenoids}

All of the studied rock samples illustrate unimodal normal alkane distribution patterns, typically ranging from $n-\mathrm{C}_{15}$ to $n$ - $\mathrm{C}_{34}$ (Fig. 9). Normal alkanes less than $\mathrm{C}_{14}$ are absent, probably because of evaporative loss during sample preparation. The maximum peak is generally detected in the range from $n-\mathrm{C}_{17}$ to $n-\mathrm{C}_{27}$. This normal alkane distribution pattern is characteristic of source rocks with strong input from marine organic matter. The pristane to phytane ratio $(\mathrm{Pr} / \mathrm{Ph})$ is considered as an indicator of the redox condition of the depositional environment. Low $\mathrm{Pr} / \mathrm{Ph}$ ratios $(<1)$ reflect an anoxic depositional environment, while greater values reveal more oxic conditions (Peters et al. 2005). In the studied samples, phytane is dominant over pristane and the $\mathrm{Pr} / \mathrm{Ph}$ ratio displays values lower than 0.6 (Table 5). These values suggest marine reducing depositional conditions which is verified by $\operatorname{Pr} / n-\mathrm{C}_{17}$ lower than 1 and $\mathrm{Ph} / n-\mathrm{C}_{18}$ greater than 0.5 (Table 5). In the crossplot of $\mathrm{Pr} / n-\mathrm{C}_{17}$ versus $\mathrm{Ph} / n-\mathrm{C}_{18}$, the studied samples fall in the zone of marine Type II kerogen deposited under reducing conditions (Fig. 10).

\subsection{Thermal maturity}

The evaluation of thermal maturity of organic matter in the studied samples was carried out using vitrinite reflectance $\left(\% R_{\mathrm{o}}\right)$, pyrolysis $T_{\max }$, and production index (PI) values. All of the Rock-Eval $S_{2}$ values are greater than $0.5 \mathrm{mg} \mathrm{HC/g}$ rock (Tables $1,2,3,4$ ), so $T_{\max }$ is reliable for thermal maturity evaluation (Tissot and Welte 1984). The measured vitrinite reflectance values are in good agreement with pyrolysis $T_{\max }$ data (Fig. 11). The mean vitrinite reflectance values of the studied samples are in the range of $0.35-0.82 \% R_{\mathrm{O}}$ (Tables 1 , $2,3,4)$ showing thermally immature to mature stage of hydrocarbon generation in the analyzed samples. This is supported by $T_{\max }$ and PI values in the range of $402-464{ }^{\circ} \mathrm{C}$ and $0.03-0.35$, respectively (Tables $1,2,3,4$ ). Thermal maturity has also been estimated by $\mathrm{HI}-T_{\max }$ plot which indicates that the studied samples contain immature to late mature organic matter (Fig. 8a). The lowest vitrinite reflectance, $T_{\max }$, and PI values were recorded in the central wells, while the highest values were measured for the samples from the eastern wells (Tables 1, 2, 3, 4). Generally, the Kazhdumi Formation and Ahmadi Member with older age and deeper burial are more thermally mature than the Pabdeh and Gurpi formations.

\subsection{Burial and thermal history modeling}

To generate reliable burial and thermal history models, the accurate timing and duration of erosional events should be fully constrained. As a consequence of eustatic sea-level changes and epeirogenic movements, several regional unconformities, erosion, and hiatuses occurred through the sedimentary succession of the study area (Sharland 2001). 

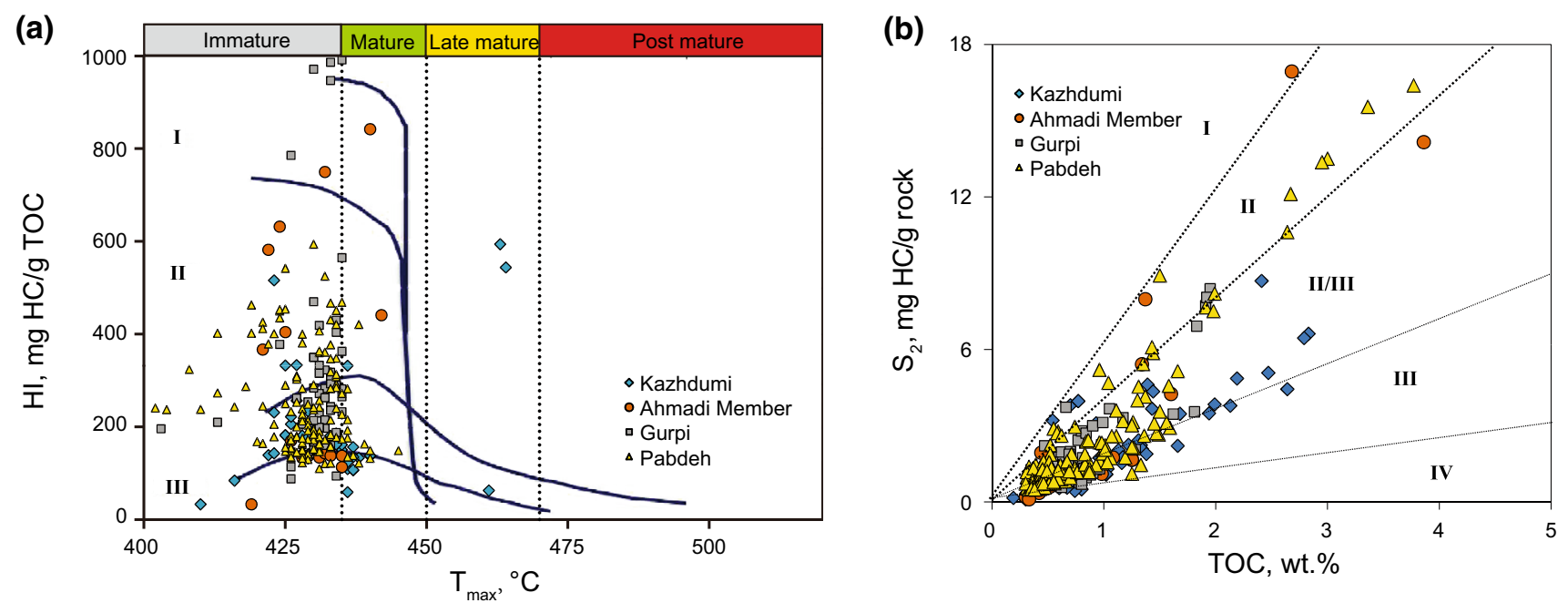

Fig. 8 a Modified Van Krevelen diagram of HI versus $T_{\max }$ and $\mathbf{b} S_{2}$ versus TOC for the analyzed samples

In the Mid-Late Cretaceous and then in the Cenozoic, hiatus and erosion affected the studied area as a consequence of collision of the Arabian and Eurasian plates forming the Zagros Mountains (Zagros orogeny). The widespread Turonian unconformity occurred in the study area as a result of localized uplift (following the initiation of ophiolite obduction along the northeastern plate margin) and a global eustatic fall in sea level. The upper part of the Sarvak Formation is sometimes absent due to erosion during the Turonian unconformity. The pre-Neogene sediments are one of the most important erosional surfaces in the Tertiary sequences in the study area. Tectonics and eustasy integrated to cause a major relative sea-level fall through the Oligocene time resulting in widespread erosion and non-deposition across the entire region (Ghazban 2009). In general, total erosions between 50 and $200 \mathrm{~m}$ were considered in our models (Table 6). However, after carrying out a comprehensive sensitivity analysis and trying out several scenarios, the amount of erosions was found to have negligible impact on the present-day maturity and temperature trends. The results of the best fit models are presented here.

\subsubsection{Well P-1, within the Field P}

In well P-1, a constant heat flow value of $63 \mathrm{~mW} / \mathrm{m}^{2}$ (from the Late Cretaceous onward) gives the best fit between the measured and calculated vitrinite reflectance and bottomhole temperatures (Fig. 12a). Compared to other wells, the studied source rocks have attained higher levels of maturity in the well P-1 possibly because of the deeper burial. The Ahmadi Member and Kazhdumi Formation, with maximum burial depths greater than $4000 \mathrm{~m}$, are at the late oil window in this well with maximum burial temperatures of 152 and $160{ }^{\circ} \mathrm{C}$ and calculated vitrinite reflectance values of $1.2 \% R_{\mathrm{o}}$ and $1.25 \% R_{\mathrm{o}}$, respectively (Figs. 13, 14). The onset of the oil window $\left(0.55-0.7 \% R_{\mathrm{o}}\right)$ was in the Early Eocene (49 Ma) at a depth of approximately $1800 \mathrm{~m}$ (Fig. 12a). Within the Middle-Late Eocene (38 Ma) and at a depth greater than $2500 \mathrm{~m}$, these source rocks entered the main oil window $\left(0.7-1 \% R_{\mathrm{o}}\right)$, and during the Late Miocene time $(8 \mathrm{Ma})$, they reached the late oil window $\left(1-1.3 \% R_{\mathrm{o}}\right)$. The transformation ratio (TR), which is defined as the ratio of generated hydrocarbons to the total generation potential of a source rock (Shalaby et al. 2011), reached $96 \%$ for the Kazhdumi Formation and Ahmadi Member (Figs. 13, 14). The Gurpi Formation has just reached the late oil window with a maximum burial temperature of $143{ }^{\circ} \mathrm{C}$ and calculated vitrinite reflectance of $1 \% R_{\mathrm{o}}$ (Figs. 12a, 15). Hydrocarbon generation begun from the late Middle Eocene (40 Ma) at a burial depth of approximately $1800 \mathrm{~m}$. Main oil generation occurred during the Late Oligocene (24 Ma) at a depth of approximately $2400 \mathrm{~m}$. The Pabdeh Formation with a maximum burial temperature of about $124{ }^{\circ} \mathrm{C}$ and calculated vitrinite reflectance of $0.8 \% R_{\mathrm{o}}$ is interpreted to be within the main oil generation window (Figs. 12a, 16). Oil generation began in the Oligocene (30 Ma) at a burial depth of 


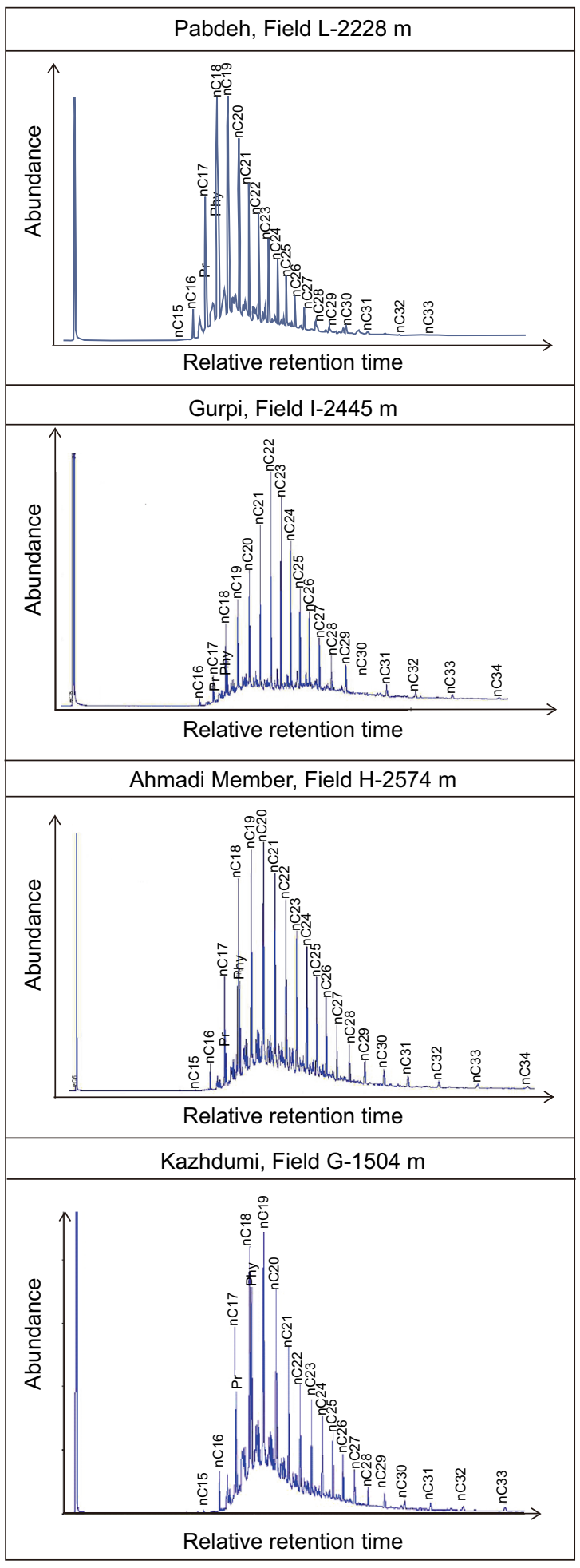

Fig. 9 Examples of gas chromatograms for the selected samples

approximately $1800 \mathrm{~m}$ and the main oil window occurred in the Late Miocene (7 Ma). The TR reached $84 \%$ and $40 \%$ for the Gurpi and Pabdeh formations, respectively.

\subsubsection{Well J-1, within Field J}

In well $\mathrm{J}-1$, a constant heat flow value of $72 \mathrm{~mW} / \mathrm{m}^{2}$ (from the Late Cretaceous onward) gives the best fit between the measured and calculated vitrinite reflectance and bottomhole temperatures (Fig. 12b). Based on the burial/thermal history model, the Kazhdumi Formation and Ahmadi Member are in the main oil window in this well with maximum burial temperatures and calculated vitrinite reflectance values of approximately $115{ }^{\circ} \mathrm{C}$ and $0.75 \% R_{\mathrm{o}}$, respectively (Figs. 12b, 13, 14). These source rocks reached the required levels of thermal maturity for the onset of the oil window from the late Middle Eocene at burial depths greater than $1500 \mathrm{~m}$. At a burial depth greater than $2600 \mathrm{~m}$, the Kazhdumi Formation and Ahmadi Member reached the main oil generation window in the Late Miocene and Pliocene, respectively. The TR of these source rocks reached approximately $20 \%$ in this well. The Gurpi and Pabdeh formations are early mature with estimated maximum burial temperature and calculated vitrinite reflectance values of approximately $90{ }^{\circ} \mathrm{C}$ and $0.6 \% R_{\mathrm{o}}$, respectively (Figs. 12b, 15). The oil generation in the Gurpi Formation started from the Late Miocene (9 Ma) at a depth of $2100 \mathrm{~m}$. The Pabdeh Formation has just reached the required thermal maturity for the hydrocarbon generation (Figs. 12b, 16). The generated hydrocarbons by these formations are not significant with TR values lower than $7 \%$.

\subsubsection{Well F-1, within Field F}

Well F-1 is modeled with a constant heat flow value of $71 \mathrm{~mW} / \mathrm{m}^{2}$ from the Late Cretaceous onward. This value led to a good match between the measured and calculated vitrinite reflectance and bottom-hole temperatures (Fig. 17a). All of the studied source rocks are thermally immature in this well, with estimated maximum burial temperatures lower than $80{ }^{\circ} \mathrm{C}$ and calculated vitrinite reflectance values lower than $0.5 \% R_{\mathrm{o}}$ (Figs. 13, 14, 15, 16).

\subsubsection{Well D-1, within Field D}

In well D-1, the best fit between the calculated and measured vitrinite reflectance and bottom-hole temperatures was obtained assuming a constant heat flow of $60 \mathrm{~mW} / \mathrm{m}^{2}$ for the Lower Cretaceous onward (Fig. 17b). In this well, the Pabdeh Formation is not present and the facies change to the Jahrum Formation. Other studied rock units are immature in this well with estimated maximum burial temperatures lower than $80{ }^{\circ} \mathrm{C}$ and calculated vitrinite reflectance lower than $0.5 \% R_{\mathrm{o}}$ (Figs. 13, 14, 15, 16). 


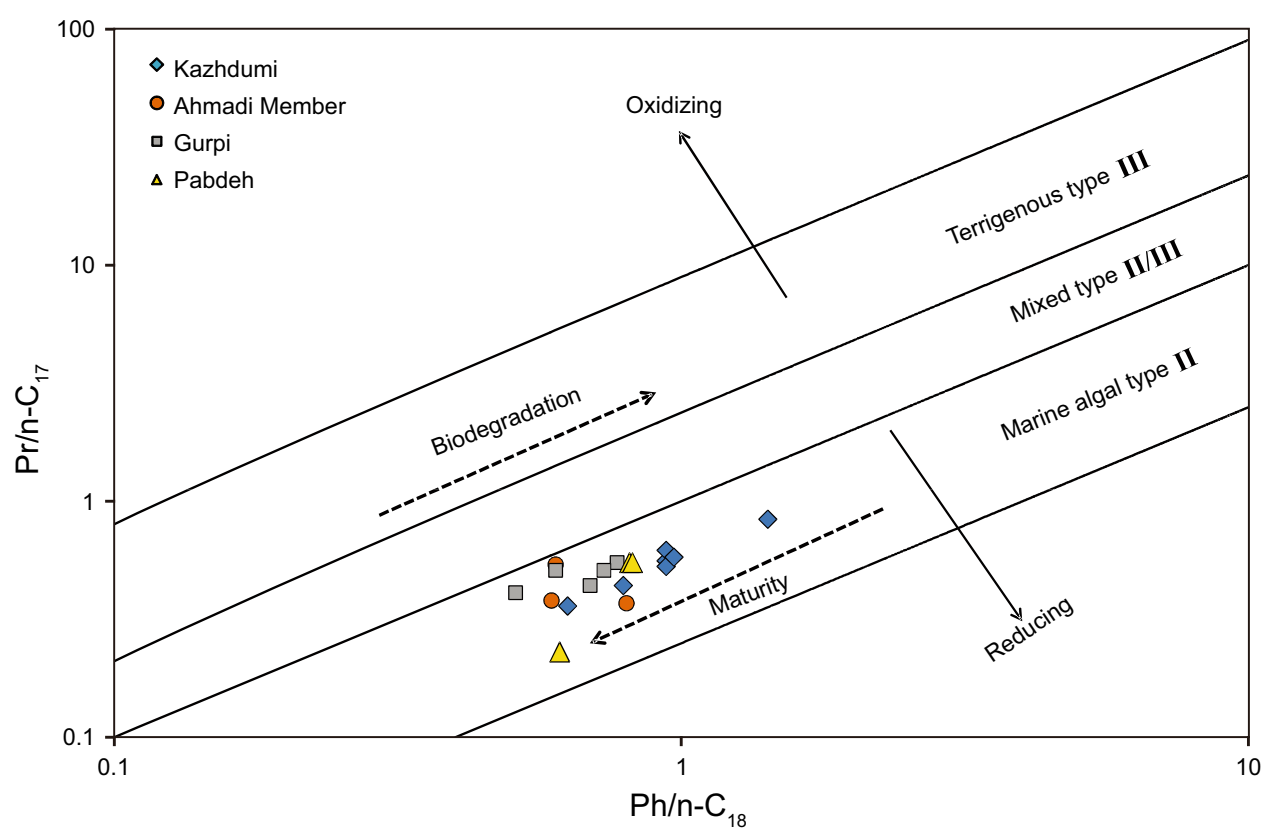

Fig. 10 Cross-plot of $\operatorname{Pr} / n-C_{17}$ versus $\mathrm{Ph} / n-\mathrm{C}_{18}$ for the studied crude samples

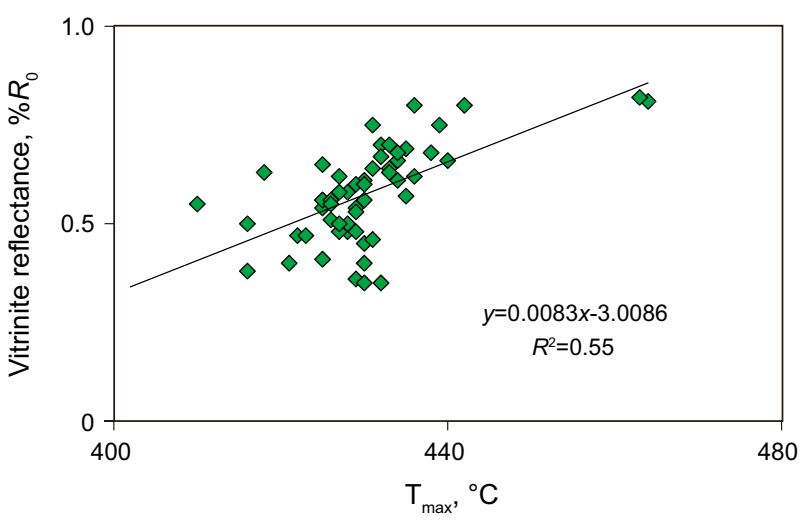

Fig. 11 Plot of $T_{\max }$ versus vitrinite reflectance for the studied samples

\subsubsection{Well C-1, within Field C}

In well C-1, a constant heat flow value of $73 \mathrm{~mW} / \mathrm{m}^{2}$ (from the Late Cretaceous onward) gives the best fit between the measured and calculated vitrinite reflectance and bottom-hole temperatures (Fig. 18a). The Pabdeh Formation changes to the Jahrum Formation and is not present in this well. The Kazhdumi Formation is at an early mature stage, with a maximum burial temperature of approximately $100{ }^{\circ} \mathrm{C}$ (occurred at Late Eocene) and calculated vitrinite reflectance of $0.65 \% R_{\mathrm{o}}$ (Fig. 13). The onset of the oil window was in the Middle Eocene (47 Ma) at a depth greater than $1400 \mathrm{~m}$ for this formation (Fig. 18a). The generated hydrocarbon of the Kazhdumi Formation is not significant in this well location with TR lower than $10 \%$ (Fig. 13). The Ahmadi Member and Gurpi Formation are thermally immature with maximum burial temperatures lower than $85{ }^{\circ} \mathrm{C}$ and calculated vitrinite reflectance lower than $0.5 \% R_{\mathrm{o}}$ (Figs. 14, 15).

\subsubsection{Well B-1, within Field B}

Well B-1 was modeled with a constant heat flow value of $72 \mathrm{~mW} / \mathrm{m}^{2}$ from the Late Cretaceous onward. With this value both the temperature and maturity trends have a reasonably good fit with the observed data (Fig. 18b). The Kazhdumi Formation and Ahmadi Member are interpreted to be early mature, with maximum burial temperatures of 106 and $97{ }^{\circ} \mathrm{C}$ and calculated vitrinite reflectance values of $0.67 \% R_{\mathrm{o}}$ and $0.62 \% R_{\mathrm{o}}$, respectively (Figs. 13, 14). The onset of oil generation from the Kazhdumi Formation and Ahmadi Member occurred in the Oligocene (at a burial depth of $1500 \mathrm{~m}$ ) and Late Miocene (at a depth of $1788 \mathrm{~m}$ ), respectively. TR values lower than $10 \%$ indicate that the generated hydrocarbons by these source rocks are not significant. The Gurpi and Pabdeh formations, with maximum burial temperatures lower than $90{ }^{\circ} \mathrm{C}$ and calculated vitrinite reflectance lower than $0.5 \% R_{\mathrm{o}}$, are thermally immature (Figs. 15, 16).

As discussed previously, the studied source rocks have fair hydrocarbon generation potential in the study area. In source rocks with fair generation potential, the necessary 

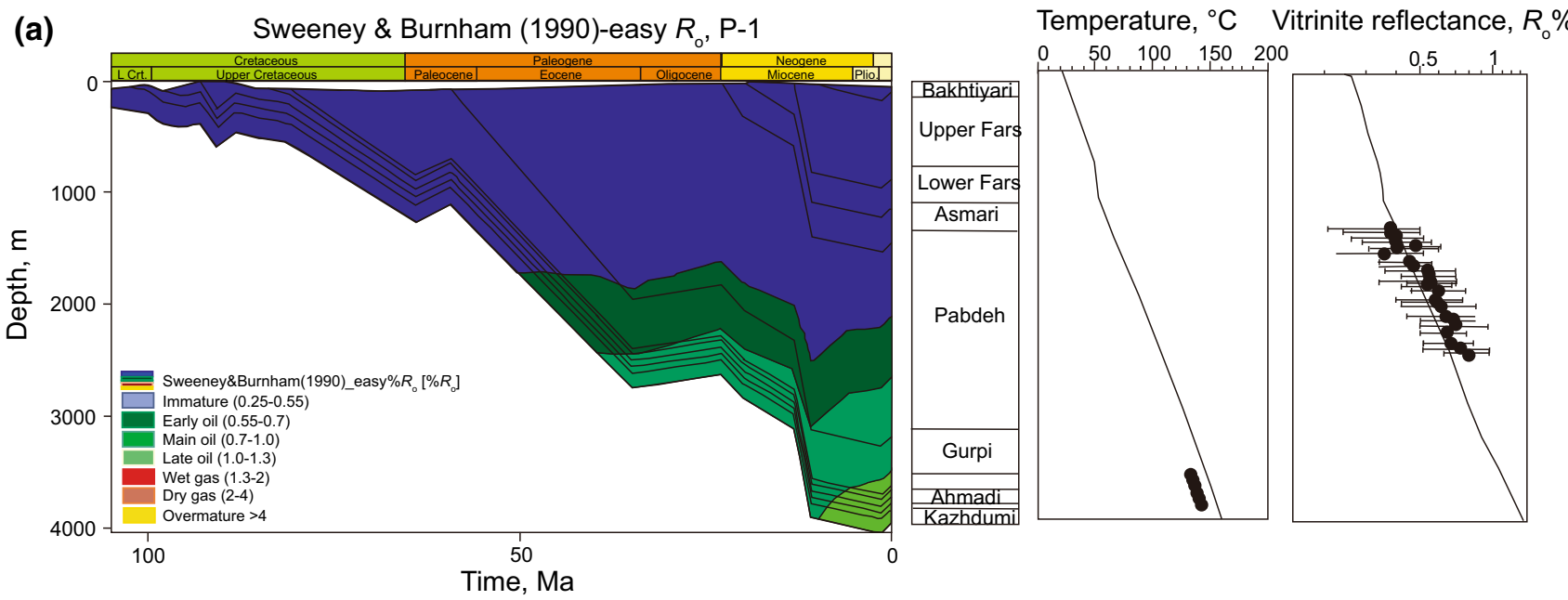

(b)

Sweeney \& Burnham (1990)-easy $R_{\mathrm{o}}, \mathrm{J}-1$
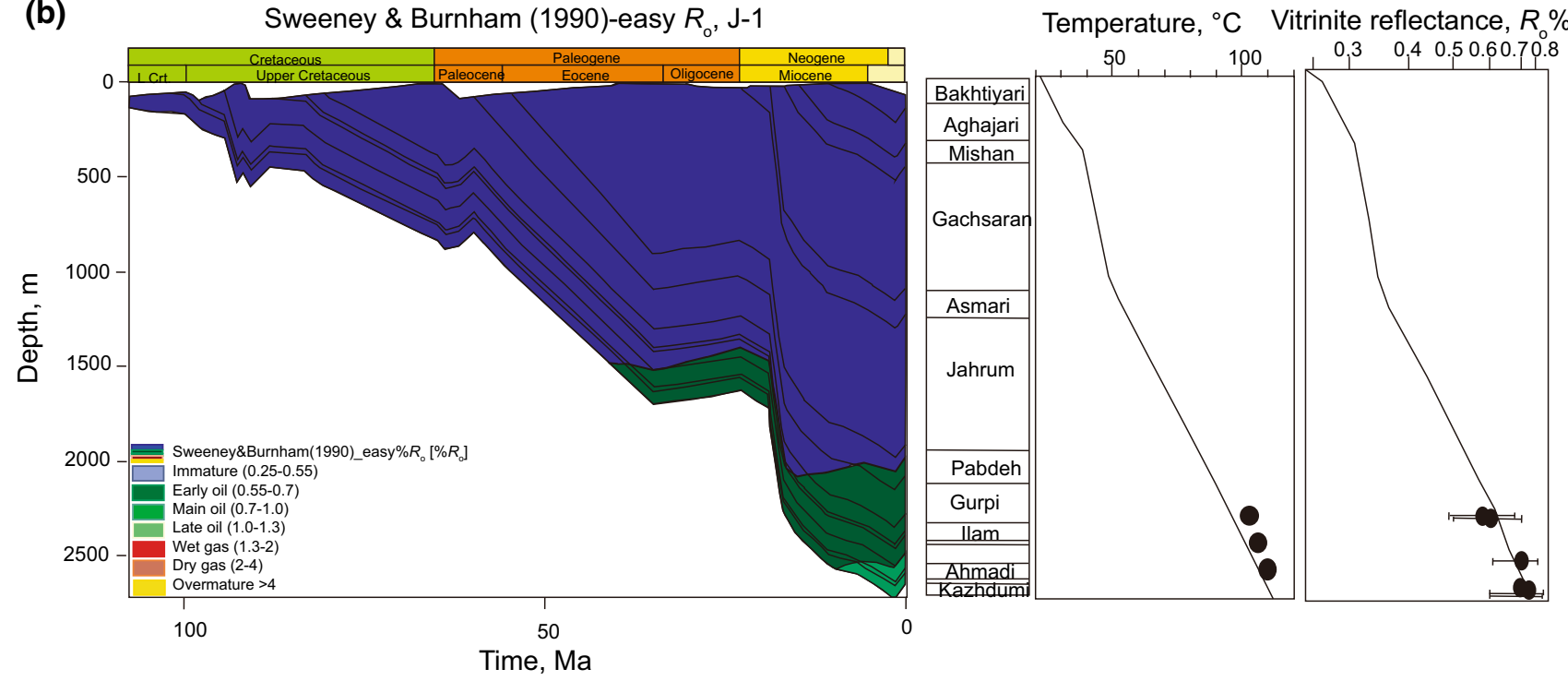

Fig. 12 Burial and thermal modeling of the eastern wells P-1 (top) and J-1 (bottom). The calibrations of the measured and calculated bottomhole temperature and vitrinite reflectance data are also shown

TR required to reach the onset of expulsion was estimated to be in the range of $45 \%-55 \%$ (Bordenave and Hegre 2010). Only in the well P-1, are the TR values of the Kazhdumi Formation, Ahmadi Member, and Gurpi Formation in the required range for hydrocarbon expulsion when start of the expulsion is in the Early to Late Miocene.

Due to shallower depth of burial and younger age, the Gurpi and Pabdeh formations generally have lower thermal maturity than the Kazhdumi Formation and Ahmadi Member in the study area. All of the studied source rocks are immature in the central wells. The presence of the Qatar Arch and distribution of Hormoz Salt in the Persian Gulf region can be possible reasons for lower thermal maturity in the central parts. The Qatar Arch has deformed the sedimentary cover by an order of magnitude more than the diapiric structures. A basement high is thus inferred in the core of the Qatar Arch and it has separated the Persian Gulf into northwest and southeast parts (Konert et al. 2001). The presence of this paleohigh at the central part of the Persian Gulf caused different burial depths for the studied rock units in the region such that they have shallower burial depth around the Qatar Arch, while being more deeply buried in the surrounding areas (Alsharhan and Nairn 1997). Lower thermal maturity of the studied formations in the central part can be a result of this lower burial depth in this part of the study area (Fig. 4).

Salt has a thermal conductivity two to four times greater than that of other sedimentary rocks (Bjørlykke 2010). It can have a great impact on the maturity of the organic matter and timing of hydrocarbon generation. Because the 

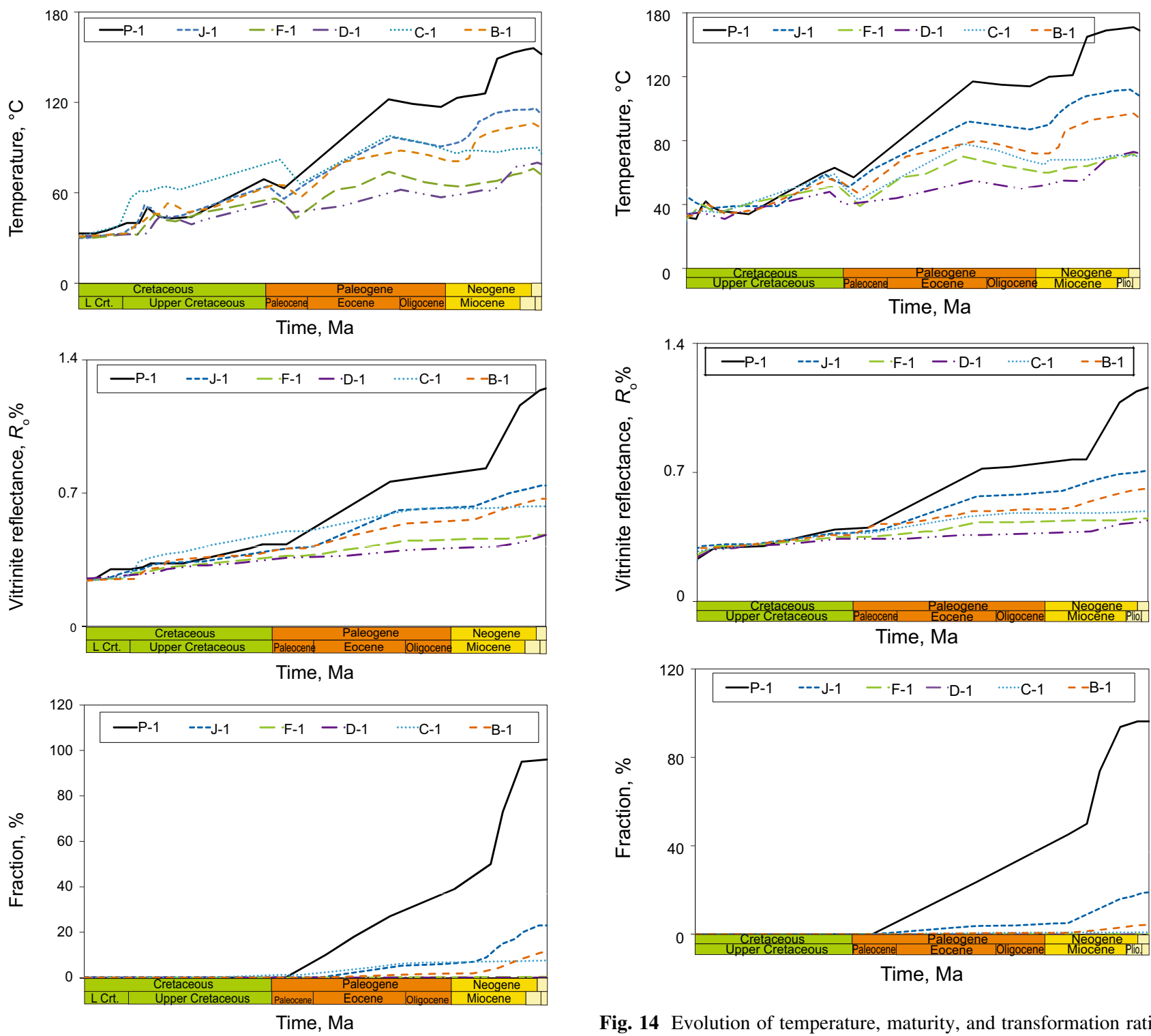

Fig. 13 Evolution of temperature, maturity, and transformation ratio (TR) for the Kazhdumi Formation in the investigated wells of the study area

heat is transferred more easily to the source rock units situated above the salt layers, they become more mature compared to adjacent source rocks not affected by salt. The salt diapirs of the Late Proterozoic Hormoz Formation in the area are present only in southeast and northwest of the Qatar Arch, while they are absent around the crest of this arch (Husseini 2000) (Fig. 3). The absence of salt-related phenomena around the crest of the arch is possibly due to the lack or reduced thickness of the Hormoz Formation in this region (Konert et al. 2001). So the presence of the Hormoz Formation in the southeast and northwest of the Qatar Arch can be considered as another possible reason

Fig. 14 Evolution of temperature, maturity, and transformation ratio (TR) for the Ahmadi Member in the investigated wells of the study area

for higher thermal maturity in these areas relative to the central part influenced by the Qatar Arch.

\section{Summary and conclusions}

The Kazhdumi Formation, the Ahmadi Member of the Sarvak Formation, and the Gurpi and Pabdeh formations were introduced as source rock candidates of the Middle Cretaceous-Early Miocene petroleum system in the Persian Gulf. In this study, hydrocarbon generation potential, depositional environment, and thermal maturity of 262 

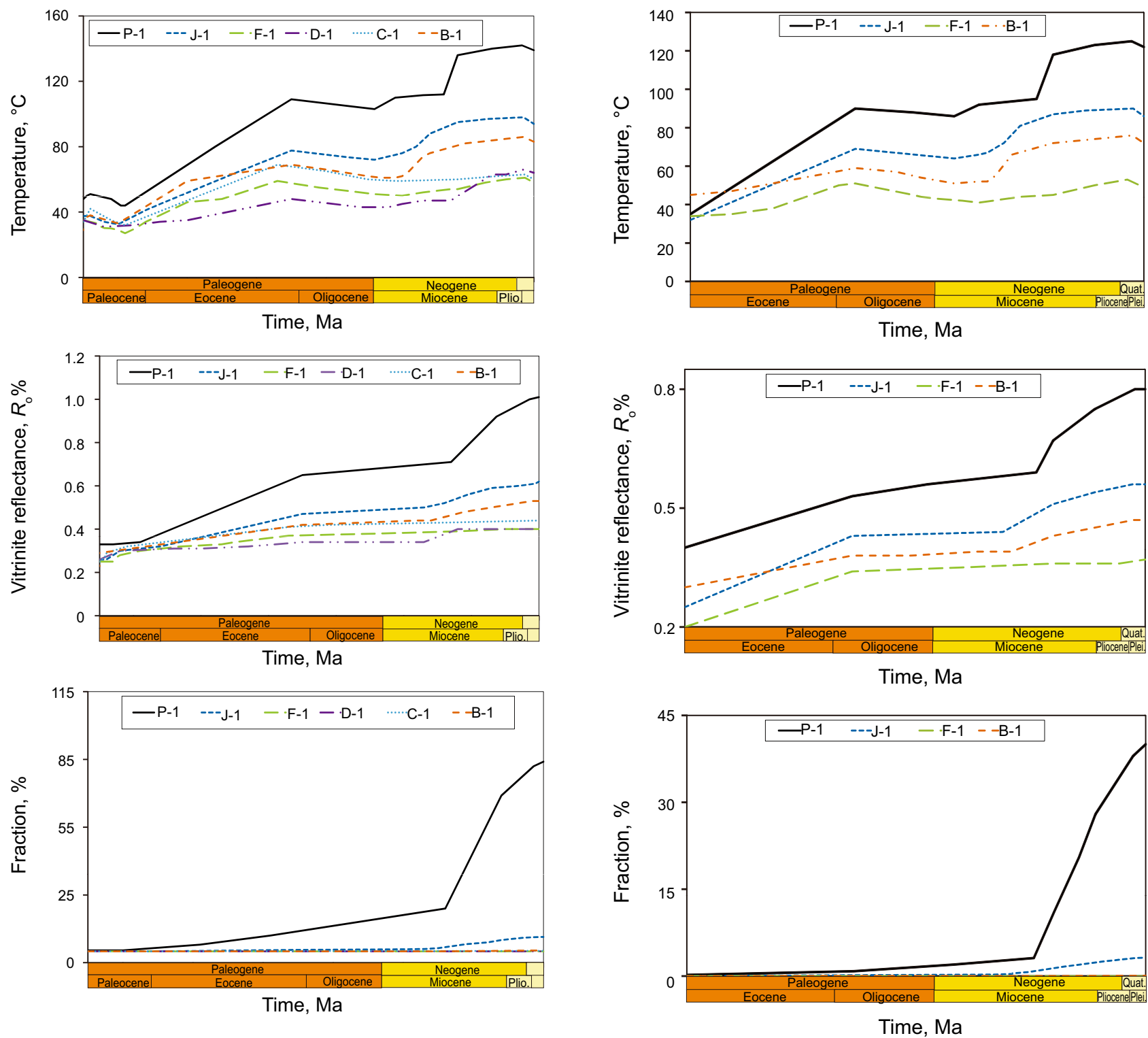

Fig. 15 Evolution of temperature, maturity, and transformation ratio (TR) for the Gurpi Formation in the investigated wells of the study

Fig. 16 Evolution of temperature, maturity, and transformation ratio (TR) for the Pabdeh Formation in the investigated wells of the study area

cutting samples of these rock units were analyzed in 16 fields located in the Iranian sector of the Persian Gulf. Also, by using PetroMod 1D software, burial and thermal histories were modeled for six selected wells in the study area to analyze the thermal maturity evolution and hydrocarbon generation histories of the Kazhdumi Formation, Ahmadi Member, and the Gurpi and Pabdeh formations. Bottom-hole temperatures and measured vitrinite reflectance values were used for calibration of models.

- Based on Rock-Eval pyrolysis data and normal alkane distribution patterns, the studied source rock candidates

have fair hydrocarbon generation potential and deposited under marine reducing conditions with marine organic matter as the main input.

- Vitrinite reflectance, Rock-Eval pyrolysis $T_{\max }$, and PI values indicate a wide range of maturities between thermally immature to mature for the studied rock units. The Kazhdumi Formation is more thermally mature than the other source rocks. The highest level of maturity is observed in the eastern parts of the study area.

- The constant heat flow values in the range of 63-73 (from the Late Cretaceous onward) give the best fit 


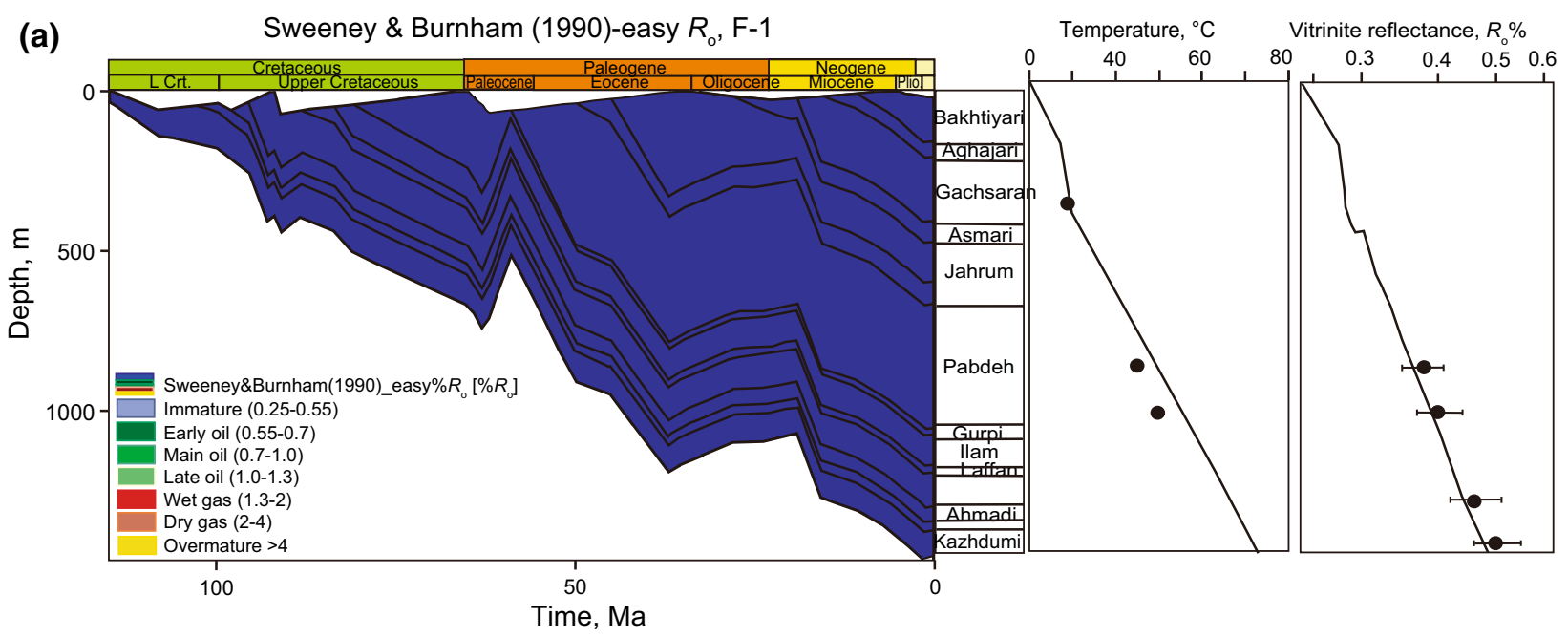

(b) Sweeney \& Burnham (1990)-easy $R_{0}$, D-1

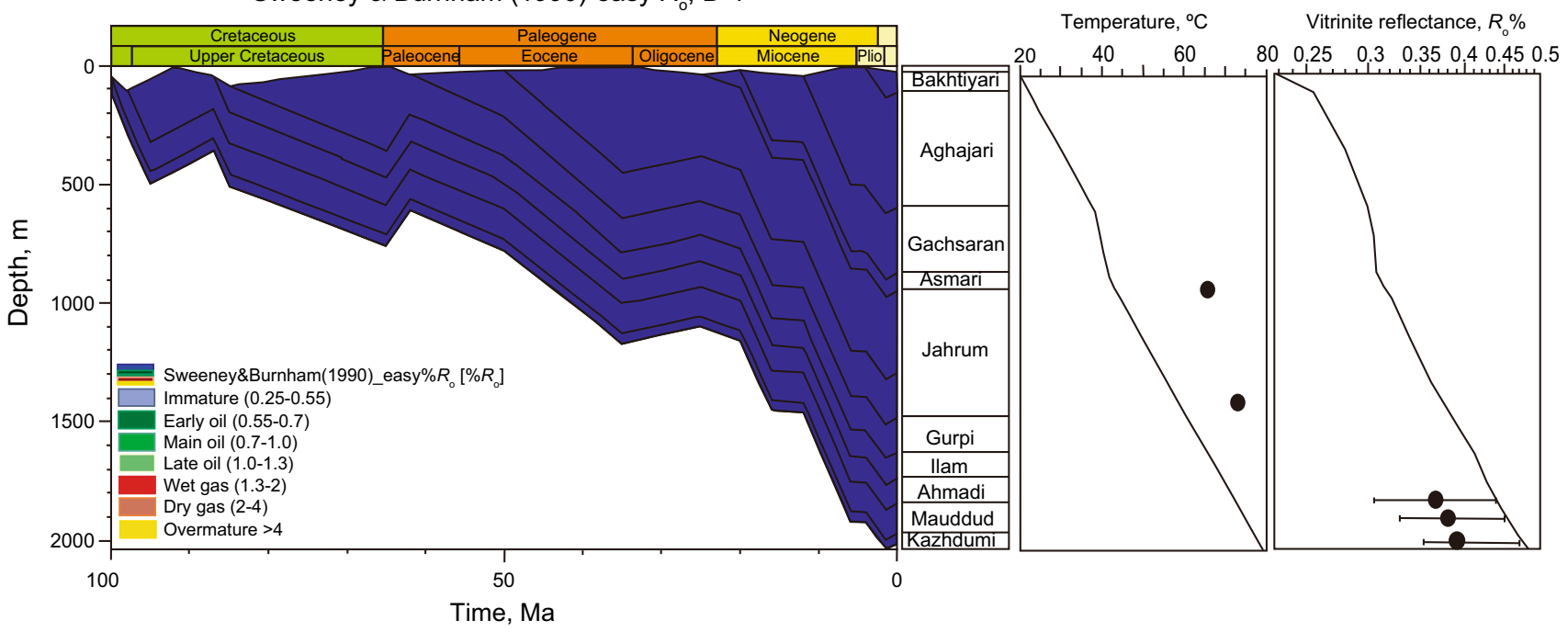

Fig. 17 Burial and thermal modeling of the central wells F-1 (top) and D-1 (bottom). The calibrations of the measured and calculated bottomhole temperature and vitrinite reflectance data are also shown

between the measured and calculated bottom-hole temperatures and vitrinite reflectance values in the studied wells.

- The studied source rock candidates are not sufficiently mature for hydrocarbon generation in the central wells (D-1 and F-1).

- The Kazhdumi Formation is early mature in the western wells (B-1 and C-1) and is in the main oil window in the eastern wells (J-1 and P-1). The hydrocarbon generation from the Kazhdumi Formation started from the Early Eocene, whereas the main phase of generation begun during Late Miocene.

- The results of the burial and thermal modeling indicate that the Ahmadi Member is immature in well C-1 and it is early mature in the B-1 well. In the eastern wells (P-1 and $\mathrm{J}-1$ ), the Ahmadi Member is in the main oil window. The oil generation from the Ahmadi Member may have begun from Early Eocene and the main oil window occurred in the Late Miocene.

- The Pabdeh and Gurpi formations are thermally immature for hydrocarbon generation in the western wells. They are early mature in well $\mathrm{J}-1$ and are in the main oil window in well P-1. The hydrocarbon generation from the Gurpi Formation started in the Middle Eocene and the main phase of oil generation was in the Late Oligocene. The onset of oil generation from the Pabdeh Formation was in the Oligocene and the main oil window was within the Late Miocene.

- Due to the higher thermal maturity, the Kazhdumi Formation and Ahmadi Member probably have a more significant role in charging the reservoirs of the study area than the Gurpi and Pabdeh formations. 

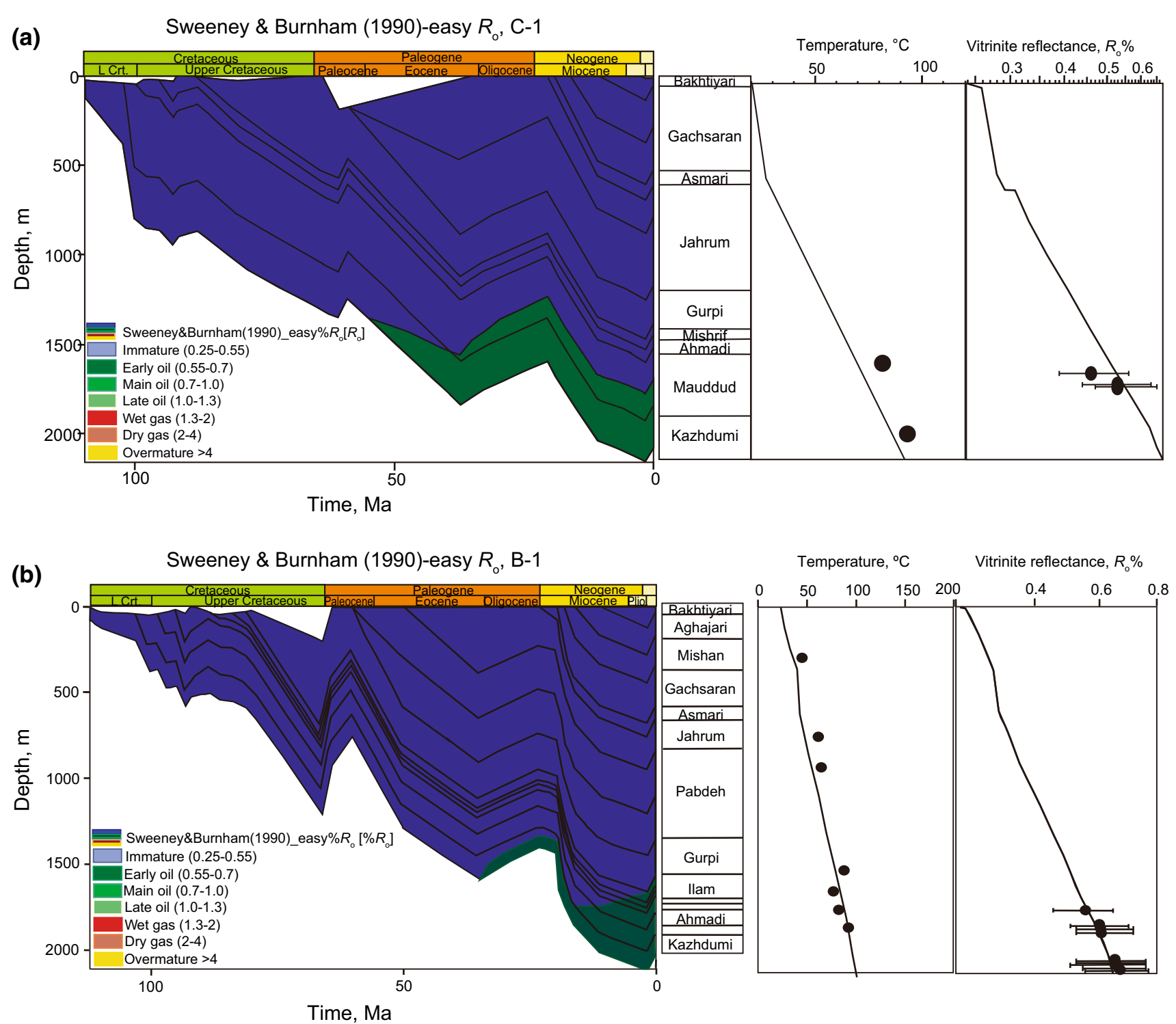

Fig. 18 Burial and thermal modeling of the central wells C-1 (top) and B-1 (bottom). The calibrations of the measured and calculated bottomhole temperature and vitrinite reflectance data are also shown

- The onset of oil expulsion in the studied source rocks was after deposition of related cap rocks, which allows the accumulation of generated hydrocarbons in the available reservoirs.

Acknowledgments The authors thank the Research Institute of Petroleum Industry (RIPI) and especially Dr. Mohammad Reza Kamali for providing facilities to complete this study.

Open Access This article is distributed under the terms of the Creative Commons Attribution 4.0 International License (http://creativecommons.org/licenses/by/4.0/), which permits unrestricted use, distribution, and reproduction in any medium, provided you give appropriate credit to the original author(s) and the source, provide a link to the Creative Commons license, and indicate if changes were made.

\section{References}

Aali J, Rahimpour-Bonab H, Kamali MR. Geochemistry and origin of the world's largest gas field from Persian Gulf, Iran. J Pet Sci Eng. 2006;50(3-4):161-75.

Alavi M. Regional stratigraphy of the Zagros fold-thrust belt of Iran and its proforeland evolution. Am J Sci. 2004;304(1):1-20.

Al-Husseini MI. Launch of the Middle East geological time scale. GeoArabia. 2008;13(4):185-8.

Alizadeh B, Sarafdokht H, Rajabi M, Opera A, Janbaz M. Organic geochemistry and petrography of Kazhdumi (Albian-Cenomanian) and Pabdeh (Paleogene) potential source rocks in southern part of the Dezful Embayment, Iran. Org Geochem. 2012;49:36-46.

Alsharhan AS, Kendall CGSC. Cretaceous chronostratigraphy, unconformities and eustatic sealevel changes in the sediments 
of Abu Dhabi, United Arab Emirates. Cretac Res. 1991;12(4):379-401.

Alsharhan AS, Nairn AEM. Sedimentary basins and petroleum geology of the Middle East. Amsterdam: Elsevier; 1997.

Bjørlykke K. Petroleum geoscience from sedimentary environments to rock physics. Berlin: Springer; 2010.

Bordenave ML. The Middle Cretaceous to Early Miocene petroleum system in the Zagros Domain of Iran, and its Prospect Evaluation. Presented at the Annual Meeting of American Association of Petroleum Geologists, Houston, Texas; 10-13 March 2002.

Bordenave ML, Burwood R. Source rock distribution and maturation in the Zagros Orogenic Belt: provenance of the Asmari and Bangestan Reservoir oil accumulations. Org Geochem. 1990;16(1-3):369-87.

Bordenave ML, Hegre JA. Current distribution of oil and gas fields in the Zagros Fold Belt of Iran and contiguous offshore as the result of the petroleum systems. Geol Soc Lond Spec Publ. 2010;330(1):291-353.

Bordenave ML, Huc AY. The Cretaceous source rocks in the Zagros foothills of Iran. Oil Gas Sci Technol-Rev IFP. 1995;50(6):727-52.

Burnham AK. A simple kinetic model of petroleum formation and cracking. Lawrence Livermore National Laboratory Report UCID-21665 1989.

Edgell HS. Proterozoic salt basins of the Persian Gulf area and their role in hydrocarbon generation. Precambrian Res. 1991;54(1):1-14.

Espitalié J, Laporte JL, Madec M, Marquis F, Leplat P, Paulet J, et al. Méthode rapide de caractérisation des roches mètres, de leur potentiel pétrolier et de leur degré d'évolution. Oil Gas Sci Technol-Rev IFP. 1977;32(1):23-42.

Ghasemi-Nejad E, Head MJ, Naderi M. Palynology and petroleum potential of the Kazhdumi Formation (Cretaceous: AlbianCenomanian) in the South Pars field, northern Persian Gulf. Mar Pet Geol. 2009;26(6):805-16.

Ghazban F. Petroleum geology of the Persian Gulf. Tehran: Tehran University Press; 2009.

Ghazban F, Al-Aasm IS. Hydrocarbon induced diagenetic dolomite and pyrite formation associated with the Hormoz Island Dome, Offshore Iran. J Pet Geol. 2010;33:183-96.

Gradstein FM, Ogg JG, Smith A, et al. Geologic timescale 2004. Cambridge: Cambridge University Press; 2004.

Haghi AH, Kharrat R, Asef MR, Rezazadegan H. Present-day stress of the central Persian Gulf: implications for drilling and well performance. Tectonophysics. 2013;608:1429-41.

Homke S, Vergés J, Serra-Kiel J, Bernaola G, Sharp I, Garcés M, et al. Late Cretaceous-Paleocene formation of the proto-Zagros foreland basin, Lurestan Province, SW Iran. Geol Soc Am Bull. 2009;121(7-8):963-78.

Hunt JM. Petroleum geochemistry and geology. New York: W.H. Freeman; 1996.

Husseini MI. Origin of the Arabian plate structures: amar collision and Najd rift. GeoArabia. 2000;5(4):527-42.

Kent PE. The emergent Hormuz salt plugs of southern Iran. J Pet Geol. 1979;2(2):117-44.

Konert G, Afifi AM, Al-Hajri SA, Droste HJ. Paleozoic stratigraphy and hydrocarbon habitat of the Arabian Plate. GeoArabia. 2001;6(3):407-42.

Lafargue E, Marquis F, Pillot D. Rock-Eval 6 applications in hydrocarbon exploration, production, and soil contamination studies. Oil Gas Sci Technol-Rev IFP. 1998;53(4):421-37.

Mohsenian E, Fathi-Mobarakabad A, Sachsenhofer RF, AsadiEskandar A. 3D basin modelling in the Central Persian Gulf, offshore Iran. J Pet Geol. 2014;37(1):55-70.
Murris RJ. Middle East; stratigraphic evolution and oil habitat. AAPG Bull. 1980;64(5):597-618.

Opera A, Alizadeh B, Sarafdokht H, Janbaz M, Fouladvand R, Heidarifard MH. Burial history reconstruction and thermal maturity modeling for the Middle Cretaceous-Early Miocene petroleum system, southern Dezful Embayment, SW Iran. Int J Coal Geol. 2013;120:1-14.

Perotti CR, Carruba S, Rinaldi M, Bertozzi G, Feltre L, Rahimi M. The qatar-south fars arch development (Arabian Platform, Persian Gulf): insights from seismic interpretation and analogue modelling. In: Schattner U, editor. New frontiers in tectonic research - at the Midst of Plate convergence. Croatia: InTech; 2011. p. 325-52.

Peters KE, Walters CC, Moldowan JM. The biomarker guide. Cambridge: Cambridge University Press; 2005.

Rabbani AR. Petroleum geochemistry, offshore SE Iran. Geochem Int. 2007;45(11):1164-72.

Rabbani AR. Geochemistry of crude oil samples from the Iranian sector of the Persian Gulf. J Pet Geol. 2008;31(3):303-16.

Rabbani AR. Petroleum geology and geochemistry of the Persian Gulf. Tafresh: Tafresh University; 2013.

Rabbani AR, Kotarba MJ, Baniasad AR, Hosseiny E, Wieclaw D. Geochemical characteristics and genetic types of the crude oils from the Iranian sector of the Persian Gulf. Org Geochem. 2014;70:29-43.

Rabbani A, Tirtashi RB. Hydrocarbon source rock evaluation of the super giant Ahwaz oil field, SW Iran. Aust J Basic Appl Sci. 2010;4(5):1-14.

Rahmani O, Aali J, Junin R, Mohseni H, Padmanabhan E, Azdarpour A, Zarza S, Moayyed M, Ghazanfari P. The origin of oil in the Cretaceous succession from the South Pars oil layer of the Persian Gulf. Int J Earth Sci. 2013;102:1337-55.

Rahmani O, Aali J, Mohseni H, Rahimpour-Bonab H, Zalaghaie S. Organic geochemistry of Gadvan and Kazhdumi formations (Cretaceous) in South Pars field, Persian Gulf, Iran. J Pet Sci Eng. 2010;70(1-2):57-66.

Shalaby MR, Hakimi MH, Abdullah WH. Geochemical characteristics and hydrocarbon generation modeling of the Jurassic source rocks in the Shoushan Basin, north Western Desert, Egypt. Mar Pet Geol. 2011;28(9):1611-24.

Sharland PR. Arabian plate sequence stratigraphy. Manama: Gulf PetroLink; 2001.

Soleimani B, Bahadori AR, Meng F. Microbiostratigraphy, microfacies and sequence stratigraphy of upper cretaceous and paleogene sediments, Hendijan oilfield, Northwest of Persian Gulf, Iran. Nat Sci. 2013;5(11):1165-82.

Sweeney J, Burnham AK. Evaluation of a simple model of vitrinite reflectance based on chemical kinetics. AAPG Bull. 1990;74(10):1559-70.

Taylor GH, Teichmüller M, Davis A, Diessel CFK, Littke R, Robert P. Organic petrology. Berlin: Gebrüder Borntraeger; 1998.

Tissot BP, Welte DH. Petroleum formation and occurrence. New York: Springer; 1984.

Wygrala BP. Integrated study of an oil field in the Southern Po Basin, Northern Italy. Zentralbibliothek d Kernforschungsanlage. 1989;2313:1-217.

Yalçin MN, Littke R, Sachsenhofer RF. Thermal history of sedimentary basins. In: Welte D, Horsfield B, Baker D, editors. Petroleum and basin evolution. Berlin: Springer; 1997. p. 71-167.

Ziegler MA. Late Permian to Holocene paleofacies evolution of the Arabian Plate and its hydrocarbon occurrences. GeoArabia. 2001;6(3):445-504. 